\title{
Contribution of structural connectivity to MEG functional connectivity
}

\author{
Anirudh Wodeyar ${ }^{\mathrm{a}, \mathrm{b}, \mathrm{d}}$, Ramesh Srinivasan ${ }^{\mathrm{a}, \mathrm{c}}$ \\ ${ }^{a}$ Department of Cognitive Sciences, University of California, Irvine, California \\ ${ }^{b}$ Department of Statistics, University of California, Irvine, California \\ ${ }^{c}$ Department of Biomedical Engineering, University of California, Irvine, California \\ ${ }^{d}$ Department of Mathematics and Statistics, Boston University, Boston
}

\begin{abstract}
Structural connectivity by axonal fiber bundles provides the substrate for transmission of action potentials across the brain. Functional connectivity in MEG signals is expected to arise from communication along structural connections. However, very little empirical evidence has been obtained to support this hypothesis. The main objective of this study is to use simulations and MEG data to directly evaluate the contribution of structural connectivity to MEG functional connectivity measures. Since axonal transmission is on a millisecond time scale we hypothesize that measures sensitive to phase synchronization in a frequency band, such as coherence, would have a closer relationship to structural connectivity than measures sensitive to slower time scales such as amplitude-envelope correlation. We estimate graphical models of MEG functional connectivity, i.e, the MEG effective connectivity, to reduce the influence of leakage effects and common input effects, and to explicitly model the contribution of structural connectivity to functional connectivity. Consistent with our hypothesis, networks defined by models of gamma band $(>30 \mathrm{~Hz}$ ) coherence that incorporate phase information show the closest alignment to structural connectivity. However, at lower frequencies (1-30 Hz) there was better alignment between models of amplitude envelope correlation and structural connectivity. In simulations, summarizing network properties of graphical models using graph theoretic metrics provides a robust measure of the relationship between functional and structural connectivity that is preserved even at low signal to noise ratios. In MEG data, centrality of nodes in the gamma band networks more closely correspond to centrality of nodes in the structural networks than a
\end{abstract}


direct comparison between edge weights.

Keywords: MEG, Graphical Model, Functional Connectivity, Structural Connectivity, Effective Connectivity, Graph Theory

\section{Introduction}

Functional connectivity (FC) in macroscopic brain data (fMRI, MEG,

EEG) refers to evidence of statistical dependence between signals recorded from two different brain locations. This broad definition encompasses different measurement techniques, signal processing methods, and statistical models that emphasize different temporal and spatial aspects of the underlying brain activity.

MEG (and EEG) signals are sampled on a millisecond time scale capturing aggregate synaptic activity from populations of neurons. These neurophysiological signals have intrinsic time scales of dynamics, usually organized in terms of frequency bands that exhibit distinct behaviors in different cognitive tasks and clinical disease states [1, 2, 3, 4, 5, 6]. Functional connectivity in MEG is usually estimated within predefined frequency bands, using methods that prioritize different time scales of dynamics.

Coherence is a widely used measure of functional connectivity that measures millisecond time scale synchronization [7]. Coherence is a measure of the consistency of relative phase and relative amplitude within a frequency band between signals recorded from two distinct areas. Coherence has long been interpreted as reflecting signal transmission with consistent axonal delays along white-matter tracts [8, 9]. For instance, a consistent 45 degree phase difference between two $10 \mathrm{~Hz}$ oscillations corresponds to a consistent $12.5 \mathrm{~ms}$ delay. The communication through coherence theory [10] suggests that the brain uses consistency in phase between areas to support coordination of neural activity, as consistency in phase helps align neuronal action potentials and thereby effective communication between areas.

In the past decade, several studies have investigated the correlation of the amplitude envelope of MEG signals, a measure of functional connectivity at slower (seconds) timescales. The amplitude of a band-limited oscillatory signal only varies at time scales slower than the average period of the oscillations. Hence, amplitude correlation investigates consistency of signals at a much slower timescale on the order of 100s of milliseconds to seconds. The motivation for investigating the correlation of the intensity of the signals 
(while ignoring precise phase synchronization) is to more closely match the time scales of MEG functional connectivity to fMRI functional connectivity. Indeed many studies have shown that correlation of the slow fluctuations of the MEG amplitude envelope in different frequency bands show similarities to fMRI resting-state networks [11, 12, 13, 14] that have been shown to correlate with structural connectivity [15, 16].

One of the main objectives of this study is to directly evaluate the contribution of structural connectivity to phase and amplitude-envelope based estimates of MEG functional connectivity. Structural connectivity provides a substrate for communication over axonal fiber bundles with time delays on the order of milliseconds [8] to conduct action potentials. Thus, on theoretical grounds, we hypothesize that estimates of connectivity that emphasize millisecond time scale synchronization using phase in a frequency band, such as coherence, would have a closer relationship to structural connectivity than measures of connectivity based on amplitude alone.

Previous studies that have directly compared the relationship of MEG functional connectivity with structural connectivity have found variable results [17, 18]. One study found that group-averaged structural connectivity explains only 4\% [18] variance of MEG coherence. Another study [17], using the amplitude envelope and Spearman's rank correlation found that structural connectivity explained $20 \%$ of the MEG functional connectivity variance. Directly comparing these studies is difficult because of potential limitations in simple correlation/coherence metrics of functional connectivity. These results only suggest there may be a relationship between structural connectivity and MEG functional connectivity that requires further investigation.

We do not expect $100 \%$ correspondence between structural and functional connectivity for two reasons:

1. Network activity must vary across brain state leading to different patterns of functional connectivity. Even in the resting state, we do not expect every structural connection to express functional connectivity.

2. Common input effects are a confound in functional connectivity estimation. When two sources, A and B, without a direct structural connection receive (either simultaneously or with a delay) activity from a third source $(\mathrm{C})$, then $\mathrm{A}$ and $\mathrm{B}$ will show correlated activity and apparent functional connectivity.

Practical considerations also potentially limit the correspondence between 
structural connectivity and MEG functional connectivity. In humans, structural connectivity is only estimated from Diffusion Weighted Imaging (DWI) and is an imperfect measure, subject to its own limitations [19, 20]. Also, MEG introduces challenges for investigating the relationship between structural and functional connectivity. All MEG functional connectivity studies make use of source localization algorithms that have limitations in spatial resolution. Coherence and amplitude correlation measures are both susceptible to leakage effects, where two statistically independent and spatially distinct sources in the brain aren't distinguishable via MEG source localization due to the nature of their location and orientation resulting in spurious correlated source activity.

An accurate estimate of MEG functional connectivity that reduces the influence of common inputs and leakage effects is crucial for studies examining the nature and dynamics of brain function using MEG and may clarify the relationship between MEG functional connectivity and structural connectivity. We define Effective connectivity or model based connectivity using graphical models to estimate the precision (inverse of covariance). Normalized precision potentially offers a better connectivity estimate which is more closely aligned with the underlying structural connectivity. Normalized precision estimates the conditional dependence, i.e., the strength of statistical dependence between two sources (brain areas) after accounting for variance shared with all other sources, thereby estimating "direct" connections between two sources. Estimating conditional dependencies reduces the problem of common inputs and leakage effects that spread the same signal across multiple sources.

In this study, we estimated two different graphical models for MEG effective connectivity at distinct time scales. The complex-valued Gaussian graphical model (cGGM) for millisecond time scale functional connectivity estimates effective connectivity for complex-valued data accounting for both amplitude and phase [21]. The normalized precision estimate for cGGM is partial coherence 22] with nonzero partial coherence at an edge indicating a connection. We used the amplitude Gaussian graphical model (aGGM) for modeling connectivity from the real-valued amplitude envelope and accounts for correlated amplitude fluctuations at slower time scales [23]. The normalized precision estimate for aGGM is partial correlation with nonzero partial correlation at an edge indicating a connection. In the estimation procedure we have developed, both graphical models shared a common underlying structure based on a group averaged template of axonal connectivity 
across all sources (termed the structural connectome).

Networks estimated from graphical modeling provide a better approximation to the definition of an edge (the connection strength between two brain areas) of a brain network needed for graph theoretical analysis [24]. Examinations of the brain network structure using various graph-theoretic metrics have helped us understand brain function better (e.g., which areas are well connected? how are areas functionally organized? [25, 26, 27]) while also helping generate potential biomarkers for clinical groups [3]. Characterizing a network using graph-theoretic metrics provide robust summary statistics for connectivity that retain essential information about the structure of network connectivity. We hypothesize that summarizing network properties using graph-theoretic metrics will identify critical features of the networks estimated from the graphical models that may be more closely related to graph-theoretic metrics of structural connectivity than edge by edge comparisons.

In this paper, we examine different ways to estimate MEG networks that connect sources in the brain. First, we identify which methods of effective connectivity provide greater alignment to structural connectivity in simulations where known structural connectivity generates functional connectivity. Then, we examine in empirical data, for the first time (to our knowledge) which frequency bands and what type of MEG functional connectivity measure (phase and amplitude versus amplitude-envelope) more tightly correspond to structural connectivity. We hypothesize that partial coherence estimates from the cGGM more closely follow from structural connectivity than the partial correlation estimates from the aGGM. Additionally, we examine through simulations and MEG data if using graph theory leads to increased accuracy in summarizing structural connectivity relative to directly using the edge weights from partial coherence or partial correlation.

\section{Methods}

\subsection{Modeling Framework}

\subsubsection{Overview}

A template of brain structural connectivity is used in the simulation studies and MEG data analysis presented here. This model is based on tractography analysis of the group average template of DWI constructed from 842 subjects from the HCP 842 data set [28]. We combine these streamlines with the Lausanne parcellation [29] to define 129 regions of interest (ROI) and a 
structural connectivity model. The Lausanne parcellation was also used to define a source model for MEG simulations and inverse solutions.

At the core of the simulation and data analytic strategy presented here is the notion that the structural connectivity model constrains the statistics of MEG signals. We developed two multivariate Gaussian models of effective connectivity: (1) complex-valued Gaussian graphical model (cGGM), where the structural connectivity determines the precision (inverse covariance) of the complex-valued multivariate normal distribution that generates the amplitude and phase of source activity in a frequency band, and (2) amplitude Gaussian graphical model (aGGM), where the structural connectivity determines the precision of a multivariate normal distribution that generates the amplitude envelope of source activity in a frequency band. The cGGM is a generative model of coherence, as widely used in EEG and MEG studies [7, 9, 30]. The aGGM is a generative model of amplitude correlation which has been recently introduced in the MEG literature, as an analog of fMRI functional networks [11, 13, 31].
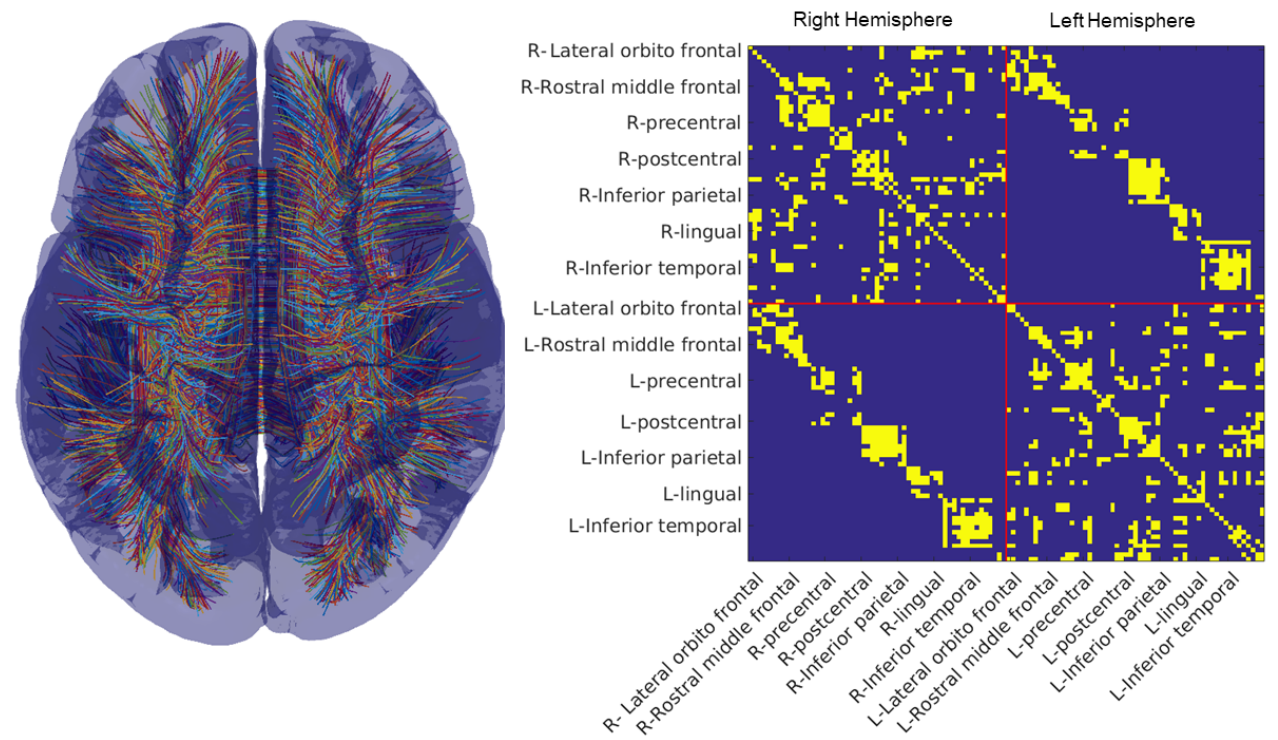

Figure 1: Structural Connectome: We show the streamlines derived from 28] on the right and the structural connectome for the 114 areas of the Lausanne parcellation on the left. We have labeled a subset of areas each with 1 to 3 subdivisions (see [29] for all subdivisions of the Lausanne parcellation). We show the binarized SC, with any non-zero edge being shown in yellow. 


\subsubsection{Structural Connectome (SC) Model}

We used streamlines generated with deterministic tractography by 28 using the HCP842 dataset 32 transformed to the MNI152 template brain obtained from FSL. We applied the Lausanne parcellation [29] of 114 cortical and 15 subcortical ROIs to the MNI152 template brain and generated a volumetric representation for each region of interest using the easy_lausanne toolbox [33]. Each streamline was approximated by a single 100-point cubic spline using code adapted from the along-tract-stats toolbox [34]. By identifying the streamlines which terminated in a pair of ROIs we were able to create the structural connectome (SC) for the Lausanne parcellation. Each streamline only connected a single pair of ROIs. Any edge $W_{i j}$ was defined as (for ROIs $i$ and $j$ connected by streamlines $s$ of length $l$ ):

$$
W_{i j}=\sum_{s_{i j}} \frac{1}{l_{i j}}
$$

We weight the streamlines by length to reduce the bias of tractography algorithms towards longer streamlines [35]. From this process, we built the $129 * 129$ weighted structural connectome with 1132 edges. We reduced this matrix to $114 * 114$ with 720 edges (see Figure 1 ) after removing all the subcortical structures and limiting inter-hemispheric connections to known homologous white-matter tracts. This latter step helped remove potentially noisy estimates of connections where streamlines intersected and passed outside the cortical surface before reaching the terminal point in a brain region. The resulting model of structural connectivity shown in Figure 1 is referred to as the structural connectome (SC). This model is incomplete in that it does not include thalamocortical projections, and there may be functional connectivity that is a result of structural connections not captured by this SC. Our estimation procedure for the graphical models of MEG functional connectivity described in the next section allows for such connections if needed to account for the statistical structure in the MEG data.

\subsubsection{Effective Connectivity: Complex-Valued Gaussian Graphical Model (cGGM)}

The template of structural connectivity derived from HCP 842[28] gives us potential constraints on the connection strengths between ROIs, and more importantly, which ROIs are not connected by white-matter tracts observable with DWI. To use this information to simulate current source activity and 
to model MEG data, we assume that the vector of source activity $(\mathbf{Z})$ at all ROIs in one frequency band is a sample drawn from a complex-valued multivariate Gaussian random process (where $\Phi$ is the precision):

$$
\mathbf{Z} \sim N(\mathbf{0}, \boldsymbol{\Phi})
$$

The band-limited MEG signal is characterized by samples of amplitude and phase, or equivalently, by analytic signal coefficients with real and imaginary parts corresponding to sine and cosine components of the signal. For frequency domain data that are complex-valued, we make use of a complexvalued multivariate normal distribution.

The complex-valued multivariate Normal for a zero-mean (where $E(\mathbf{Z})=$ 0) complex-valued Gaussian process [36] is defined as:

$$
\rho(\mathbf{Z})=\frac{1}{\pi^{n} \operatorname{det}^{\frac{1}{2}}(\boldsymbol{\Theta})} \exp \left(-\frac{1}{2} \mathbf{Z} \boldsymbol{\Theta}^{-1} \mathbf{Z}^{H}\right)
$$

where

$$
\boldsymbol{\Theta}=\left[\begin{array}{cc}
\mathbf{R}_{\mathbf{z z}} & \widetilde{\mathbf{R}}_{\mathbf{z z}} \\
\widetilde{\mathbf{R}}_{\mathbf{z z}}^{H} & \mathbf{R}_{\mathbf{z z}}{ }^{H}
\end{array}\right]
$$

and

$$
\mathbf{R}_{\mathbf{z z}}=E\left[\mathbf{z z}^{H}\right] ; \widetilde{\mathbf{R}}_{\mathbf{z z}}=E\left[\mathbf{z z}^{T}\right]
$$

The key parameter in this model is the covariance matrix $\Theta$ and its inverse, the precision matrix $\boldsymbol{\Phi}=\Theta^{-1}$. As defined in equation 4 and 5 , the covariance matrix for complex-valued data is composed of the familiar crossspectrum $\mathbf{R}_{\mathbf{z z}}$ and the complementary cross-spectrum $\widetilde{\mathbf{R}}_{\mathbf{z z}}$. Most spectral analysis methods only make use of $\mathbf{R}_{\mathbf{z z}}$ and implicitly assume circular symmetry, i.e., $\widetilde{\mathbf{R}}_{\mathbf{z z}}=0$ 36. In this case, the complex-valued data is labeled as proper. With the assumption of circular symmetry, we can parameterize the complex-valued Gaussian using the precision defined as the inverse of the cross-spectrum, $\boldsymbol{\Phi}=\boldsymbol{\Theta}^{-1}=\mathbf{R}_{\mathrm{zz}}{ }^{-1}$.

Each value in the precision matrix $\boldsymbol{\Phi}$ is the conditional covariance between any two variables (here, sources representing two ROIs) given the other variables (here, sources representing all other ROIs). The precision removes linear effects from all other sources from the covariance between any pair of sources and provides a model of functional connectivity i.e. the effective connectivity between sources. For any pair of sources, if the precision is 
zero, there is no need for a direct connection between the sources to account for observed coherence (functional connectivity). Such apparent coherences arise from connections mediated via other sources in the model.

In our model, the precision matrix $\boldsymbol{\Phi}$ captures the statistics of effective connectivity and has a nonzero entry only at edges that have a connection estimated from DWI in the SC. We are assuming that in each frequency band the connectivity we estimate arises from random fluctuations at each area becoming correlated by the SC. This generative model of the source activity giving rise to MEG signals is used in our simulation studies. In the MEG data analysis, we estimate the source precision matrix $\boldsymbol{\Phi}$ from the MEG data in each frequency band using graphical modeling techniques that inform the precision estimate with the SC estimated from the HCP-842 but allowing the precision values to be determined by the data.

\subsubsection{Effective connectivity: Amplitude Gaussian Graphical Model (aGGM)}

We use the structural connectivity to constrain potential connections due to correlated amplitude envelope fluctuations between ROIs, thereby defining the generative model of amplitude correlation. We assume that the vector of source activity amplitudes (X) at all ROIs in one frequency band is a sample drawn from a real-valued multivariate Gaussian random process (where $\Phi$ is the precision with non-zeros entries where there are $\mathrm{SC}$ connections):

$$
\boldsymbol{X} \sim N(\mathbf{0}, \boldsymbol{\Phi})
$$

When examining amplitude, we look at samples $X$ of amplitude estimates from the analytic signal of bandpass filtered MEG signal across epochs. So for amplitude data we use a real-valued multivariate normal distribution as characterizing the fluctuations of amplitudes in different frequency bands. And the multivariate normal for a zero mean, i.e., (where $E(\boldsymbol{X})=0$ ) is defined as:

$$
\rho(\boldsymbol{X})=\frac{1}{\pi^{n} \operatorname{det}^{\frac{1}{2}}(\boldsymbol{\Theta})} \exp \left(-\frac{1}{2} \boldsymbol{X} \boldsymbol{\Theta}^{-1} \boldsymbol{X}^{T}\right)
$$

This distribution is fully parameterized by the precision matrix $\mathbf{\Phi}=\Theta^{-1}$. Each value in the precision matrix $\boldsymbol{\Phi}$ is the conditional covariance between the amplitude envelope of any two sources given the other sources. The precision provides a model of amplitude envelope functional connectivity i.e the amplitude envelope effective connectivity between sources. For any pair of sources, if the precision is zero, there is no need for a direct connection between the sources to account for observed amplitude envelope correlation. 
In our model of amplitude fluctuations, the precision matrix $\boldsymbol{\Phi}$ captures the statistics of effective connectivity and has a nonzero entry only at edges (pairs of ROIs) that have a connection estimated from DWI in the SC. We are assuming that in each frequency band the connectivity we estimate arises from random fluctuations in amplitude envelopes at each area becoming correlated by the SC connections. In the MEG data analysis (both for the simulation and for real data), we estimate the source precision matrix $\boldsymbol{\Phi}$ in each frequency band using graphical modeling techniques that inform the precision estimate with the SC estimated from the HCP-842, but allowing the precision values to be determined by the data.

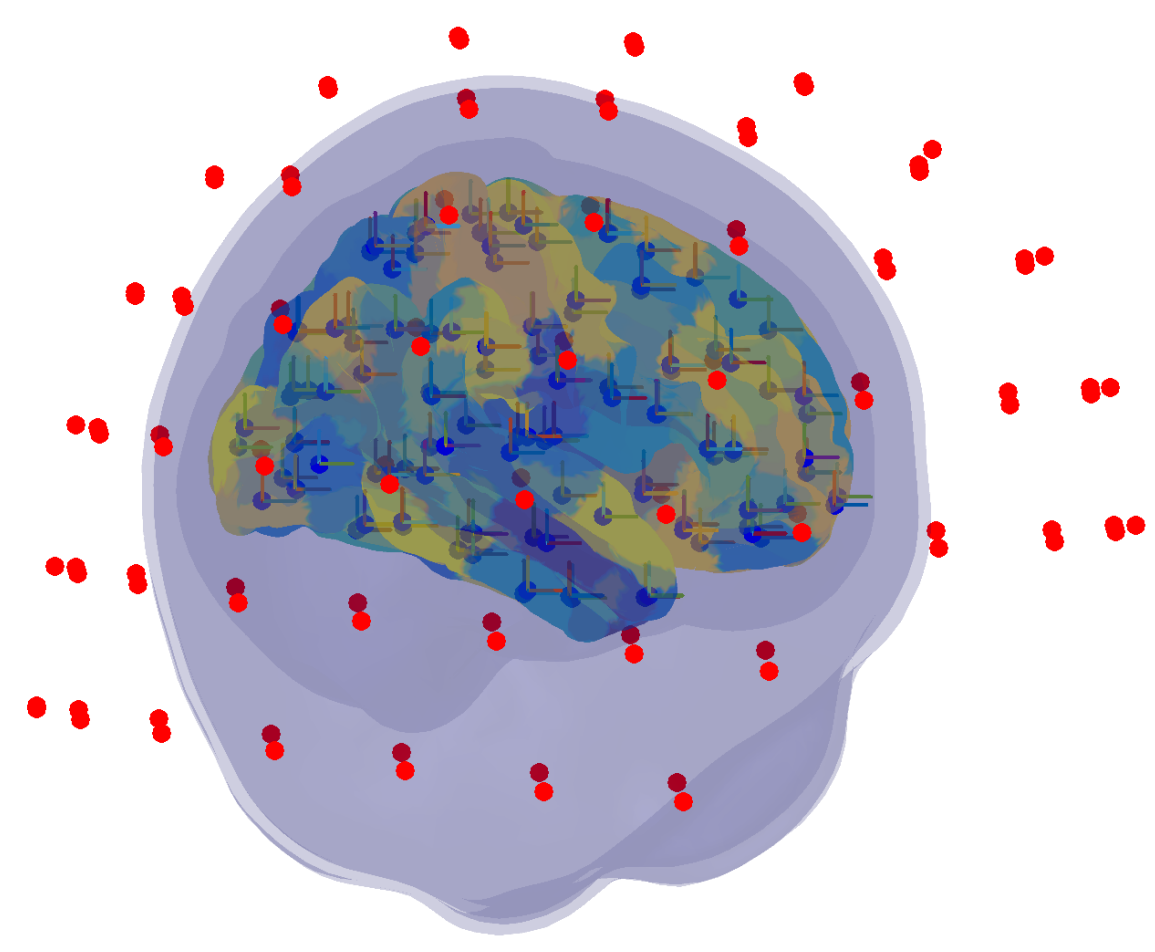

Figure 2: MEG Forward Model: We show the $f$ saverage brain subdivided into 114 regions of interest (ROIs) according to the Lausanne parcellations, with $x, y, z$ oriented dipoles representing source activity in each brain ROI. The CSF, skull and scalp boundaries obtained from the $f$ saverage head are indicated by transparent surfaces and the 102 locations of orthogonal pairs of planar gradiometers are indicated by red dots. 


\subsubsection{MEG Forward Model}

The MEG forward model is an estimate of the magnetic field measured at MEG sensors above the scalp generated by current sources located in the brain. The data analysis presented in this paper makes use of data obtained using the Neuromag MEG system consisting of 306 MEG coils at 102 locations above the scalp (shown in Figure 2). At each location, there are 3 sensors - one magnetometer that measures the component of the magnetic field passing through the coil and two planar gradiometers that measure the gradient of this magnetic field in two orthogonal directions. For both the simulations and the data analysis presented in this paper, we made use only of the orthogonal pair of planar gradiometer coils (102 pairs of sensors at 102 locations). As planar gradiometer coils have higher spatial resolution than magnetometer coils [37] we did not consider data from magnetometer coils for this study.

The head model was developed from the fsaverage MRI images from the Freesurfer toolbox [38]. The tessellated cortical surfaces for right and left hemisphere were extracted using the recon-all pipeline in Freesurfer and then downsampled to 81000 (81k) vertices (mris_decimate from Freesurfer). We used this surface to constrain dipole orientation and define the volume of the model corresponding to the cortex. We generated the inner skull, outer skull and scalp surfaces approximated with 2562 vertices from the fsaverage head generated using the mri_water shed function. Using these surfaces, and with the conductivities of the scalp, CSF and brain set at $1 \mathrm{~S} / \mathrm{m}$ and the skull at $0.025 \mathrm{~S} / \mathrm{m}$ (i.e., 40 times lower conductivity), we used the OpenMEEG toolbox [39] to compute a Boundary Element Model (BEM) to generate the MEG forward matrix. Each row of the MEG forward matrix is the magnetic field gradient detected across all 204 gradiometers from a unit current density source at one of the $81 \mathrm{k}$ cortical surface vertices.

Using the Lausanne parcellation for 114 cortical ROIs [29], we divided the cortical surface and identified vertices belonging to each ROI using the volumetric parcellation of the fsaverage brain. Using this organization of vertices we then reduced the representation of the current source for each ROI down to a set of 3 dipoles in the $x, y$, and $z$ directions at a single location. The location of the source for each ROI was selected by taking a weighted average of vertex locations where the weight of each location was determined by the magnitude (L2 norm) of the field generated at the gradiometers. In this way, we reduced our source model to 114 source locations, with 3 sources 
at each location in the canonical x,y, and $\mathrm{z}$ directions. We computed a new MEG forward matrix $(\boldsymbol{M})$ of dimension $204 * 342$ using OpenMEEG. This MEG forward matrix approximates the linear mixing of source activity at the gradiometers to generate the measured MEG signals, and will be used in generating the inverse solution discussed in the section on estimation of the model (section 2.2.2).

\subsection{Estimation of Effective Connectivity}

\subsubsection{Overview}

The estimation of the complex-valued Gaussian graphical model or the amplitude Gaussian graphical model from the MEG data proceeds in three steps. First, MEG data is bandpass filtered into each frequency band of interest. Second, an adaptive inverse solution is applied to estimate the source activity in each frequency band. After the inverse solution is applied, the Hilbert Transform is used to estimate amplitude and phase of the signals (analytic signal). We average the analytic signal over a second to define our samples of data. Two minutes of data over all sources represented an ensemble of 120 samples of analytic signal data. We used four such ensembles for each participant in all our MEG data analyses, and matched this data size in our simulations. Third, graphical modelling methods are used to estimate the precision matrix representing the Gaussian graphical model (effective connectivity). Building graphical models involves the choice of regularization parameters and we selected these parameters using cross-validation. The estimation procedure is tested through a set of simulation studies discussed in section 2.3 .

\subsubsection{Inverse Solution to estimate source activity from $M E G$}

We can represent each sample of the MEG data within a frequency band, after applying a band-pass filter, as a vector $\boldsymbol{B}$ of length equal to the number of MEG sensors (204 sensors). The set of samples of $\boldsymbol{B}$ relates to source activity $\boldsymbol{S}$ of length equal to the number of MEG sources by:

$$
B=M S
$$

where $\boldsymbol{M}$ is the MEG forward matrix. For each participant, we localized activity to the 342 sources (3 directions - along $x, y$ and $z$ axes at 114 locations) by inverting the reduced lead field using regularized minimum 
norm estimation (weighted L2 norm [40]) and applying it to data at the scalp. We estimated the inverse $\boldsymbol{M}^{-}$using (where $\nu$ is a penalization):

$$
\begin{aligned}
& \operatorname{diag}(\boldsymbol{W})=\|\boldsymbol{M}\|_{2}^{.5} \\
& \boldsymbol{M}^{-}=(\boldsymbol{M} \boldsymbol{W})^{T}\left((\boldsymbol{M} \boldsymbol{W})^{T} *(\boldsymbol{M} \boldsymbol{W})+\nu I\right)^{-1}
\end{aligned}
$$

The estimated source activity $\boldsymbol{S}=\boldsymbol{M}^{-} \boldsymbol{B}$. We identify the time series for the three dipoles along the $x, y$, and $z$ directions. Using a singular value decomposition at each ROI we identified the optimal orientation of the dipole as the first singular vector. Using the first singular vector at each ROI, we reduced the MEG forward matrix from 204x342 to $204 \times 114$. This reduced forward matrix was the individual and frequency band specific (adaptive) forward matrix. We calculated weighted L2 norm inverse for the 114 ROIs using the individual forward matrix.

\subsubsection{Frequency-domain transformation}

The source localized data at each ROI is Hilbert transformed to yield the analytic signal $\boldsymbol{A}$ which is complex-valued (instantaneous amplitude and phase). We reduced $\boldsymbol{A}$ by averaging either $\boldsymbol{A}$ over each second of data to get a sample of complex-valued data that captures the amplitude and phase of the signals or $|\boldsymbol{A}|$ (absolute value of A) to get a sample of the real-valued amplitude data. We used 120 samples across 114 ROIs to represent an ensemble of data.

\subsubsection{Adaptive Graphical Lasso using SC-QUIC}

The graphical lasso [41] is a method that has been applied in multiple fields in the past decade, from genomics [42], to fMRI functional connectivity [43, 44] and climate models [45]. This is a method that can be used to identify a sparse approximation to the precision matrix. In this way, estimates in the precision are more robust for the values that are retained. To apply the lasso, we use the penalized likelihood function as follows [46] to estimate the precision (where $\Theta$ is the covariance or cross-spectral density):

$$
\hat{\mathbf{\Phi}}=\operatorname{argmin}_{\boldsymbol{\Phi} \succ 0}\left(-\log (\operatorname{det} \boldsymbol{\Phi})+\operatorname{tr}(\boldsymbol{\Theta} \boldsymbol{\Phi})+\lambda \sum_{j<k}\left|\boldsymbol{\Phi}_{j k}\right|\right)
$$

The penalization parameter $\lambda$ in the graphical lasso determines the important set of cGGM or aGGM precision values. The output of the lasso from 
Equation 11 is the precision matrix $\hat{\boldsymbol{\Phi}}$, which represents directly the complex Gaussian Graphical (cGGM) or amplitude Gaussian Graphical model.

Our purpose was to use the lasso to estimate the precision in a way that takes advantage of our prior knowledge of the SC which are the likely locations of nonzero precision values. We made use of the lasso optimization from QUIC 47 using a matrix penalty term (this process is also called the adaptive lasso - [48]) determined by the SC with edges $W\left(\right.$ and $\lambda_{1}=\lambda_{2}$ ):

$$
\begin{aligned}
& \hat{\boldsymbol{\Phi}}=\operatorname{argmin}_{\boldsymbol{\Phi} \succ 0} \\
& \left(-\log (\operatorname{det} \boldsymbol{\Phi})+\operatorname{tr}(\boldsymbol{\Theta} \boldsymbol{\Phi})+\lambda_{1} * \sum_{j<k ; W_{j k} \in S C}\left|\boldsymbol{\Phi}_{j k}\right|+\lambda_{2} * \sum_{j<k ; \boldsymbol{W}_{j k} \notin S C}\left|\boldsymbol{\Phi}_{j k}\right|\right)
\end{aligned}
$$

Note that in the limiting case of $\lambda_{1}=\lambda_{2}$ we are using the same likelihood as the graphical lasso. Past work along these lines [23] has used the SC weights directly to determine the penalization weighting, which potentially imposes more structure than appropriate since we expect effective connectivity to vary with brain states, and the $\mathrm{SC}$ weights will constrain connectivity strengths. We do not expect the SC strengths to map directly onto the effective connectivity strengths due to individual differences, and variations within individuals across brain states. Further, the SC can be expected to have different contributions across frequency bands yielding different effective connection weights. For this reason we use the binarized SC to determine the penalization structure. We estimated the penalization values $\lambda_{1}$ and $\lambda_{2}$ using cross-validation as described in the next section (section 2.2.5).

By optimizing the above penalized likelihood, we leveraged the information in the SC as a prior for our lasso estimate. The output of the lasso when optimizing Equation 12 is the cGGM/aGGM. We derive our graph $G$ with vertices $V=1,2, \ldots, 114$ and edges $W_{e s t}=G_{i j}=1, i, j \in V$ from the cGGM/aGGM based on the non-zero values in $\hat{\boldsymbol{\Phi}}$. The final precision matrix $\tilde{\boldsymbol{\Phi}}$ is estimated under the unpenalized Gaussian likelihood for the set of edges $\boldsymbol{W}_{\text {est }}$ defined by the graphical model using the function ggmFitHtf (PMTK3 toolbox [49]) which optimizes (unpenalized Gaussian log-likelihood):

$$
\tilde{\boldsymbol{\Phi}}=\operatorname{argmin}_{\Phi \succ 0 ;|\Phi|>0=G}-(\log (\operatorname{det} \Phi)+\operatorname{tr}((\Theta+\delta * I) \Phi))
$$

Since $\Theta$ (covariance) is usually rank deficient, we add a small value $(\delta)$ along 
the diagonal to make it full rank. We define delta as 0.001 times the maximum value along the upper triangle of the covariance.

\subsubsection{Cross-validation}

The adaptive graphical lasso imposes the SC onto the functional data potentially allowing for more robust reconstructions of the precision. We tested whether the adaptive graphical lasso produced estimates of the precision that show reduced error relative to applying the graphical lasso. Note that applying the graphical lasso would be equivalent to having the penalization inside and outside the $\mathrm{SC}$ be equal i.e. $\lambda_{1}=\lambda_{2}$. We estimated the appropriate value for $\lambda_{1}$ and $\lambda_{2}$ using cross-validation.

We split the MEG data (both simulation and real data) into four contiguous pieces of 120 samples each. We estimated the precision $\tilde{\Phi}_{i}$ on one segment of the data $(i)$ and estimated the deviance when using this precision as the inverse for the covariance $\boldsymbol{\Theta}_{j}$ for all the other segments $j$ of the data (and vice versa). Deviance was estimated as:

$$
D e v=\sum_{i=1: 4} \sum_{j=1: 4 ; j \neq i}\left(-\log \left(\operatorname{det} \tilde{\Phi}_{i}\right)+\operatorname{tr}\left(\Theta_{j} \tilde{\Phi}_{i}\right)\right)
$$

We found that, in the majority of cases, both in simulation and in data, using the prior of the SC reduced cross-validated deviance relative to using graphical lasso directly. In other words, we found that the minimum crossvalidated deviance mostly occurred with $\lambda_{1}<\lambda_{2}$. This is discussed in the Results.

\subsubsection{Summary Statistics - Partial Coherence and Partial Amplitude Cor- relation}

In every frequency band, or for each iteration of our simulation, we estimated two graphical models. We estimated the complex Gaussian Graphical model to estimate the precision for complex-valued data incorporating amplitude and phase and the amplitude Gaussian Graphical model for the real-valued amplitude data. For the cGGM we estimate the partial coherence $(P C)$ as a summary statistic [22]:

$$
P C_{z_{1} z_{2}}=\left|\frac{\Phi_{z_{1} z_{2}}}{\sqrt{\Phi_{z_{1} z_{1}} * \Phi_{z_{2} z_{2}}}}\right|^{2}
$$


For the aGGM we estimate the amplitude partial correlation $(A P C)$ as a summary statistic as (assuming the precision matrix $\Phi$ here is the output from estimating an aGGM):

$$
A P C_{x_{1} x_{2}}=\left|\frac{\Phi_{x_{1} x_{2}}}{\sqrt{\Phi_{x_{1} x_{1}} * \Phi_{x_{2} x_{2}}}}\right|
$$

\subsubsection{Summary Statistics - Graph Theory}

In silico we compare the accuracy of using the estimates for PC and APC relative to using summary statistics of networks derived from graph theory. We expect that graph theoretic metrics being a summary of the network potentially boosts our accuracy in estimating network characteristics. We derive our graph $G$ with vertices $V=1,2, \ldots 114$ and edges $W_{e s t}=G_{i j}=P C_{i j}$ or $A P C_{i j}, i, j \in V$ from the graphical model using the non-zero values. We estimate graph theoretic metrics using the Brain Connectivity Toolbox [50]. We make use of several measures in this study:

1. The basic measure of a network is the degree of a node (connectivity), that is, the number of edges a node shares with other nodes in an undirected graph. This measures how many nodes can communicate with any given node.

2. The local integration of functional clusters of the network is measured by the clustering coefficient. The clustering coefficient is the ratio of the number of triangles to number of triples in a network and helps give an estimate of how often nodes one degree apart are connected to one another.

$$
\begin{gathered}
C_{i}=\frac{\text { number of triangles connected to node } i}{\text { number of possible triangles centered on vertex } i} \\
C=\frac{1}{n} \sum_{i} C_{i}
\end{gathered}
$$

3. A metric that describes the global segregation of the network is the path length. Path length $(\mathrm{L})$ is the average shortest path $(d)$ between all pairs of nodes.

$$
L=\frac{1}{\frac{1}{2} n(n+1)} \sum_{i \leq k} d_{i j}
$$

4. Closeness Centrality uses the path length matrix between every pair of nodes to define centrality. The closeness centrality for a node is the 
average path from it to all other nodes in the largest connected network. It is defined as:

$$
C_{\text {Cent }}=\frac{N}{\sum_{j=1: n} L_{i j}}
$$

5. Betweenness Centrality is an estimate of how central a node is based on the number of shortest paths it participates in. A node which lies on more shortest paths in a network has a higher betweenness centrality.

6. Eigenvector Centrality is an estimate of the closeness of a node to nodes that are strongly connected to the rest of the network.

The closeness centrality and betweenness centrality can only be estimated for a connected network. Some reconstructed partial coherence and partial correlation networks weren't fully connected. When the network had multiple connected components we selected the largest connected component and estimated closeness and betweenness centrality for that component alone. In the majority of cases this component included over 100 (out of 114) sources.

\subsection{Simulations}

\subsubsection{Overview}

We simulated effective connectivity from the SC by assuming that the SC weights constrained the precision matrix for a complex-valued multivariate normal distribution. In the simulations (see Figure 3 for a graphical presentation) we generated novel networks with random weights for edges. While the edge locations are always retained, we randomized the weights on the edges by shuffling the original SC weights and generated random relative phases for each of the edges. Simulated data (480 samples across 114 sources) generated using this precision is forward modeled to MEG sensors. We added noise to the sensor data to alter signal to noise ratio for each simulation. Using the inverse solution we estimated source activity. We matched the data analysis for real data by working with 4 ensembles of 120 samples each when estimating the appropriate penalization for the adaptive graphical lasso using cross-validation. We compared estimated and original precision over a set of metrics to examine how well we recovered the precision under different signal to noise regimes.

\subsubsection{Generative Model}

The structural connectome has connection strengths between areas defined by the weighted sum of streamlines. To generate novel precision matrices for each iteration of our simulation, we retained the edge locations from 


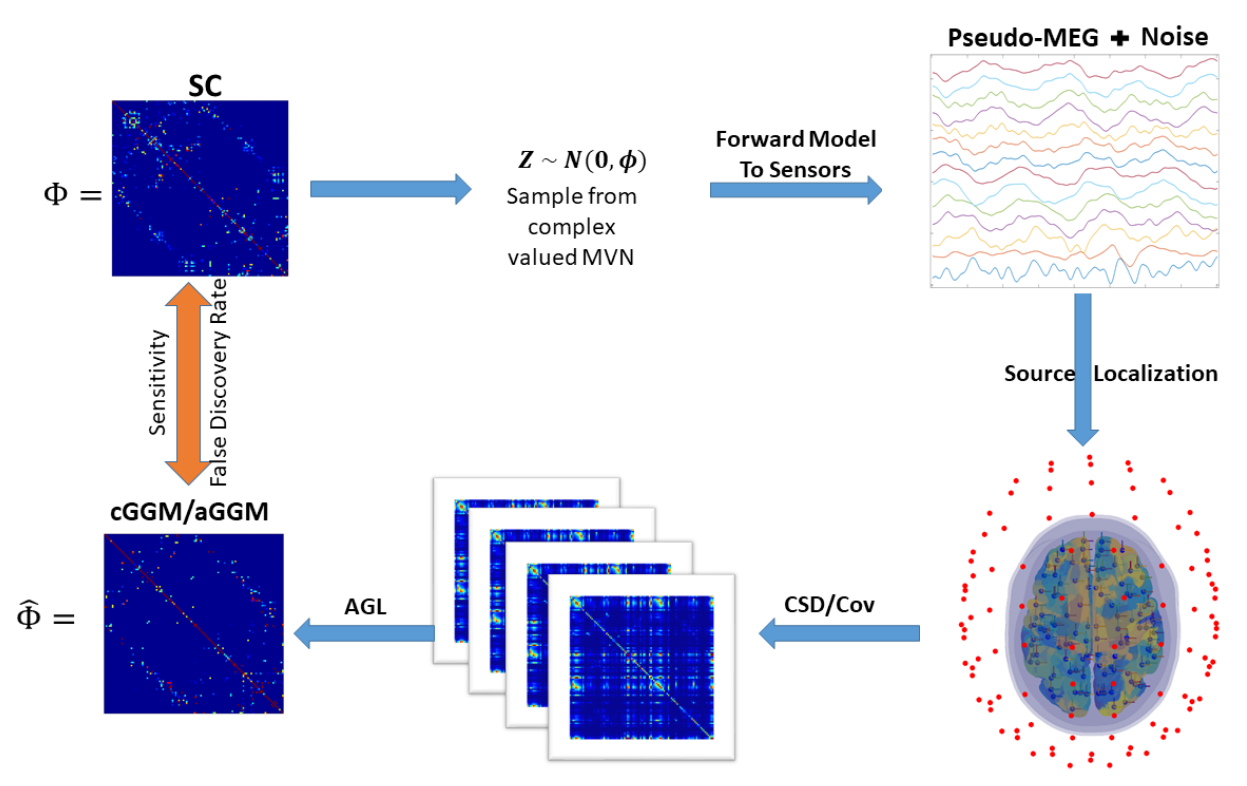

Figure 3: Simulation Overview - describing each figure above in a clockwise manner from top left: First, we used the SC edge locations and weights to constrain the precision on each iteration of the simulation. The original SC weights were shuffled across the edges and we generated random phases for each edge (described in Methods, see Eq. 22). Second, we sampled from a complex-valued multivariate normal distribution using the precision generated in the first step. Third, we used the MEG forward matrix to forward model the samples to the sensors. Fourth, we applied an inverse solution to source localize data. Fifth, we split the data into 4 ensembles of 120 samples (represented are the 4 covariance matrices from these ensembles of data). Finally, these 4 ensembles served as the input for the adaptive graphical lasso. The estimated precision from this procedure was compared to the original precision (orange arrow) using sensitivity and false discovery rate (FDR).

the original SC but randomized the original connection strengths across these locations. We permuted the connection strengths across the original set of edges. Each edge was then multiplied with a uniform random value between $\{-1,1\}$. Finally, each edge is assigned a random phase $(\mu)$ for a $10 \mathrm{~Hz}$ oscillation based on sampling from a uniform distribution for conduction velocities between $\{4,14\} \mathrm{m} / \mathrm{s}$ applied to estimated streamline lengths [8]. For a streamline of length $d$ and a conduction velocity $v$ (set to a single value for all streamlines for each simulation), we can derive the phase using: 


$$
\begin{aligned}
& t=1000 * \frac{d}{v} \\
& \mu=\bmod \left(\frac{t}{100}, 2 \pi\right)
\end{aligned}
$$

After multiplying each edge with the appropriate phase (based on average streamline lengths for that edge), we can generate the precision. This represents the complex-valued, circularly symmetric precision matrix $(\boldsymbol{\Phi})$ for a frequency band.

\subsubsection{Cross Spectral Density and Amplitude Covariance}

Using the previously defined precision, we can directly generate the cross spectral density (covariance) as its inverse $\left(\boldsymbol{\Theta}=\boldsymbol{\Phi}^{-1}\right)$. As the CSD has a realvalued equivalent [36], treating real and imaginary components as separated variables governed by the single covariance structure, we sampled $480 x 114$ complex Gaussian values using the Matlab function mvnrnd operating on the equivalent real-valued covariance matrix. The complex-valued samples are used to estimate the cross spectrum (covariance) $\hat{\Theta}$ and its inverse, the precision $\hat{\boldsymbol{\Phi}}$ ). We normalized the precision to get the partial coherence following equation 15 .

We get the amplitude estimates by taking the absolute value for all $480 x 114$ samples i.e. $\boldsymbol{X}_{a m p}=|\boldsymbol{X}|$. Using this data we estimated the original amplitude covariance and inverted it to estimate the amplitude precision matrix. The normalized amplitude precision is the amplitude partial correlation (see Eq. 16).

The estimated source partial coherence and amplitude partial correlation at this stage serve as the optimal solutions if we were directly measuring source activity. We compare all reconstructed estimates (after forward modeling data, source localization and adaptive graphical lasso) to these matrices.

\subsubsection{Forward Solution and Source Localization}

As described earlier we built our MEG forward matrix from 114 sources using the fsaverage head projecting to MEG planar gradiometers. We simulated sources of different orientations on every run of our simulation. Complexvalued data $(Z)$ is projected using this forward matrix (see Eq. 8) to the sensors. Noise is added to all sensors while controlling SNR, estimated as $(C$ is sensor covariance, and $N$ is noise covariance): 


$$
S N R=10 * \log _{10}\left(\frac{\operatorname{trace}(C)}{\operatorname{trace}(N)}\right)
$$

We examined reconstruction of networks with SNR of approximately 1, $5,10,20,30$ and $50 \mathrm{~dB}$. Source localization was performed using weighted $L^{2}$ norm inverse solutions as discussed in section 2.2.2. We generated inverse solutions across a range of penalizations and examined correlation between reconstructed cross spectrum and original source cross spectrum. We chose the optimal penalization based on the value that yielded maximum correlation between the reconstructed cross spectrum and original source cross spectrum.

\subsubsection{Estimating Partial Coherence and Partial Correlation}

After source localization, we have 480 samples of analytic signal at 114 sources. We split this data into 4 ensembles of 120 samples each in order to match the MEG data. Using this data we estimated the CSD and the amplitude covariance which served as the input to the adaptive graphical lasso. To run our SC based models, we needed to decide on two penalization values: penalization inside the SC edges $-\lambda_{1}$ and penalization outside the $\mathrm{SC}$ edges $-\lambda_{2}$. We decided these values based on cross-validation. This procedure is discussed in detail in section 2.2.5 on cross-validation.

\subsubsection{Estimating Accuracy of Network Reconstruction}

We examined reconstruction error for both sets of graphical models (partial coherence and partial correlation) and error of graph theoretic metrics. Reliability of effective connectivity reconstruction was assessed in two ways. First, we examined the sensitivity and false discovery rate in simulations since we possessed a ground truth - the SC - that gave rise to the observed covariance. Sensitivity was estimated as:

$$
S=\frac{\sum\left(W_{e s t} \in W_{S C}\right)}{\sum\left(W_{S C}\right)}
$$

Sensitivity tells us the ratio of edges recovered within the $\mathrm{SC}$ relative to the total number of SC edges. A higher value of sensitivity indicates we are able to recover more edges from the SC. Note that the relevance of using sensitivity as a measure of performance when we have biased our estimates with the SC comes from the virtue of deciding the penalization values based on cross-validation. In our procedure, it is possible for there to be an effective 
connectivity model identified by the adaptive graphical lasso that does not use the SC prior at all, i.e. $\lambda_{1}=\lambda_{2}$ is the optimal solution (minimum deviance in cross-validation).

We also examined the false discovery rate or FDR, which was defined as:

$$
F D R=\frac{\sum\left(W_{e s t} \notin W_{S C}\right)}{\sum\left(W_{e s t}\right)}
$$

FDR is the ratio of number of false positive edges estimates, i.e. edges outside the SC, relative to the total number of edges being estimated. An FDR of 0.5 indicates that, say 100 edges estimated, 50 edges were outside the SC.

We also examined reconstruction of original connection weights. We estimated the Pearson correlation between the Fisher r-to-z transformed original, sampling based estimate of the partial coherence/correlation with the Fisher r-to-z transformed reconstructed estimate over the set of SC edges as well as edges estimated $\left(W_{\text {est }} \cup W_{S C}\right)$.

Finally, we used Spearman rank correlation to correlate the different graph theory metrics estimated from the original and reconstructed networks.

\subsection{Application to $M E G$ data \\ 2.4.1. MEG Data}

The MEG data analyzed here was provided by the Cambridge Centre for Ageing and Neuroscience (CamCAN). CamCAN funding was provided by the UK Biotechnology and Biological Sciences Research Council (grant number BB/H008217/1), together with support from the UK Medical Research Council and University of Cambridge, UK. This data was obtained from the CamCAN repository (available at http://www.mrc-cbu.cam.ac. uk/datasets/camcan/, [51, 52]).

The MEG data was collected using a 306 sensor VectorView MEG system (Electa Neuromag, Helsinki). The 306 sensors consisted of 102 magnetometers and 204 planar gradiometers. As planar gradiometers have higher spatial resolution than magnetometers, we focused the analysis in this paper on the planar gradiometer data. The data were sampled at $1000 \mathrm{~Hz}$ and highpass filtered at $0.3 \mathrm{~Hz}$. This data was run through temporal signal space separation (tSSS, Taulu et al., 2005; MaxFilter 2.2, Elekta Neuromag Oy, Helsinki, Finland) to remove noise from external sources and to help correct for head movements (location of the head was continuously estimated using Head Position Indicator coils). MaxFilter was also used to remove the $50 \mathrm{~Hz}$ line noise and also to automatically detect and reconstruct noisy channels. 


\subsubsection{Spectral Analysis}

We extracted 480 seconds of resting state gradiometer data for individuals aged between 30 to 40 years of age. This yielded 91 subjects (50 male). We first applied a bandpass filter between 0.5 to $100 \mathrm{~Hz}$ and a notch filter at $50 \mathrm{~Hz}$ to remove line noise. We built elliptic filters (designed using fdesign.bandpass function in MATLAB) with stop band set to $0.5 \mathrm{~Hz}$ below and above pass band, stopband attenuation set to $100 \mathrm{~dB}$, and passband ripple set to 0.02 . Bandpass filtering was then done using the filtfilthd function in MATLAB to minimize phase distortion. We analyzed five frequency bands: delta (1-3 $\mathrm{Hz})$, theta $(4-7 \mathrm{~Hz})$, alpha $(8-13 \mathrm{~Hz})$, beta $(14-29 \mathrm{~Hz})$ and gamma $(30-80 \mathrm{~Hz})$. For each subject, and within each band we optimized the dipole orientation across 114 ROIs as described in section 2.2.2. Source localized data was Hilbert transformed and the analytic signal $A$ was computed.

We split the analytic signal $A$ into 1 second epochs so that we have a 480x1000x114 matrix for each frequency band. As input for estimating the cGGM, the complex-valued data we used was derived by averaging the original complex-valued analytic signal over each second. We split the 480 samples from 114 sources into 4 continuous epochs of 120 samples each based on the expectation that we would have robust, stationary networks estimable with 120 seconds [53]. Further, having 4 ensembles allowed for extensive cross-validation. As input for estimating the aGGM, we estimated amplitude envelope data by taking the absolute value of $A$ and averaging it over 1 second segments. We then followed the same procedure as described earlier in the section 2.2.5 on Cross Validation before estimating the summary statistics from the cGGMs as the partial coherence and from the aGGMs as the amplitude partial correlation. Thus, we had at the end of the analysis for each subject, cGGM and aGGM models across all 5 frequency bands.

\subsubsection{Sensitivity and FDR}

We examined sensitivity and FDR of the estimated cGGM and aGGM networks across different frequency bands and all subjects. There is a difference in interpretation of the sensitivity and FDR in simulations versus in real data. Sensitivity in real data indicates both whether SC connections are being used by different frequency bands and whether they're reliably estimated, while sensitivity in simulations simply indicated what edges were reliably estimated after forward modeling and source localization. For the FDR, while we know the ground truth from the SC in simulations, in real data, there is the influence of connections not included in the SC, including 
thalamocortical connections and neuromodulatory systems, which can lead to an increased "False Discovery Rate". Thus, in data the FDR can be (potentially) interpreted as a measure of the accuracy of the SC as a model rather than directly as false positives. A higher sensitivity and lower FDR indicates better alignment to the SC. Thus, using the sensitivity and FDR together in the MEG data analysis we can examine whether the SC is a good model for functional connectivity in the MEG data.

\subsubsection{Graph Theory}

In addition to the partial coherence and partial correlation, we also estimated summary statistics discussed previously - metrics from graph theory (see section 2.2.7). Using the graph theoretic metrics that were most accurately reconstructed in our simulations we categorized centrality of the cGGM and aGGM networks in real data. We estimated the median centrality across all subjects for the frequency bands with the highest sensitivity in the cGGM and aGGM networks. We compared this to the centrality estimated from the weighted SC.

\section{Results}

\subsection{Simulation}

We ran 500 iterations of our simulation and on each iteration a novel network was generated that represented the complex-valued precision in a single frequency band. This precision had non-zero edges only at SC edges. We forward modeled data sampled using the precision (480 samples) to the MEG sensors before applying the inverse solution and the adaptive graphical lasso to see the extent to which we could reconstruct the precision. We reconstructed the precision using cGGM and aGGM before estimating summary statistics. We evaluated the reconstruction performance of the full network using sensitivity (what percent of the SC edges were detected?), FDR (of total edges, how many are false positives?), correlation of reconstructed weights with the original weights, and the correlation of reconstructed network graph theory metrics with the original network characteristics.

\subsection{1. cGGM networks more sensitive to $S C$ than aGGM networks}

First, we examined the difference in penalizations applied for the SC edges $\left(\lambda_{1}\right.$ in Eq. 12) and for the non-SC edges $\left(\lambda_{2}\right.$ in Eq. 12). We found that for cGGM networks, penalization outside the SC edges was consistently higher 

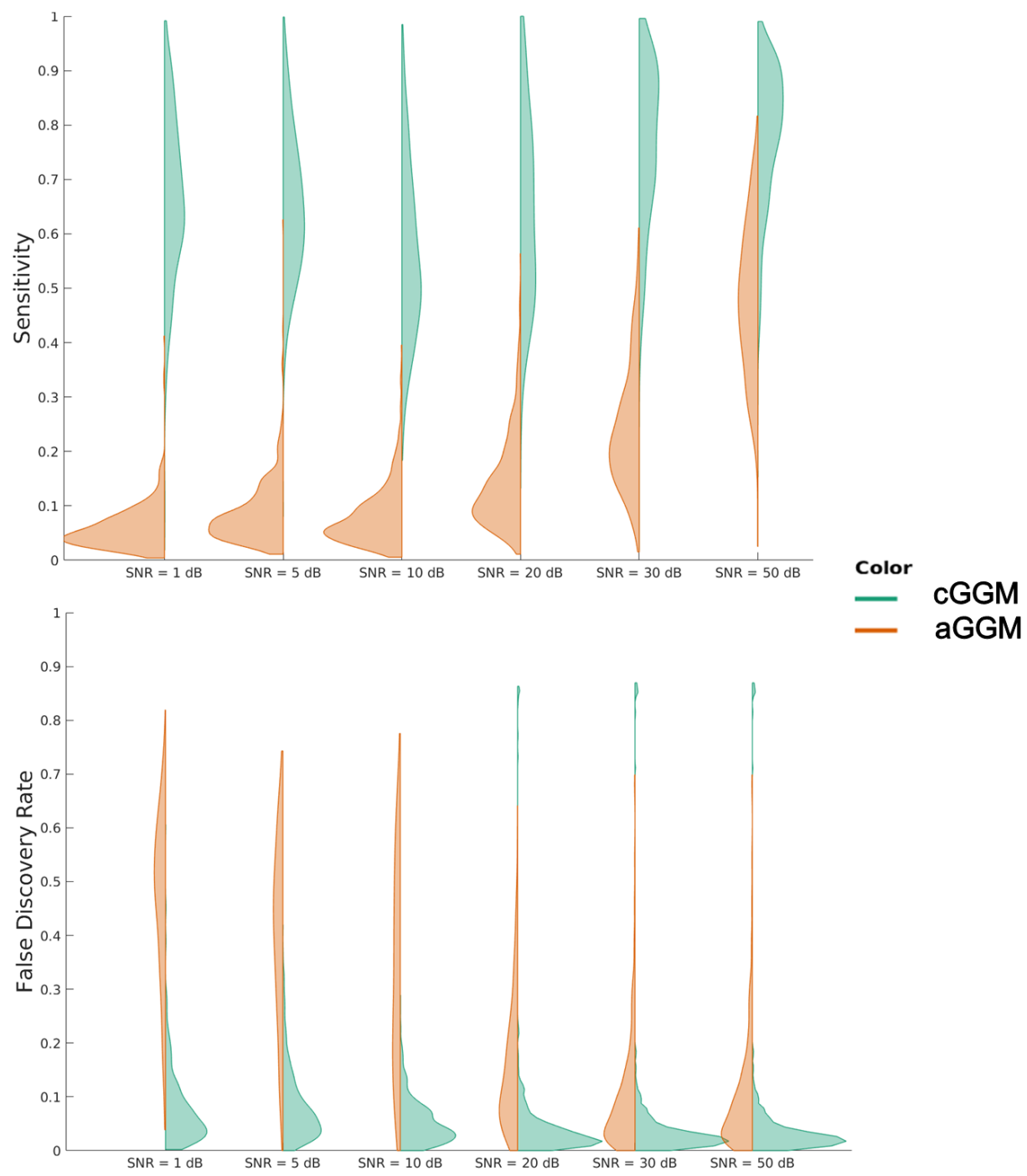

Figure 4: Sensitivity and False Discovery Rate in Simulation: We estimated networks using the cGGM and aGGM at different signal to noise ratios. We compared these networks to the binarized SC and examined the sensitivity (upper figure) and false discovery rate (lower figure). cGGM models are more sensitive to the SC across all SNR. cGGM and aGGM have comparable false discovery rates when the SNR rises above $30 \mathrm{~dB}$.

than penalization for the SC edges (i.e., $\lambda_{2}>\lambda_{1}$ ) in 2999 out of 3000 simulation iterations (across all SNR). We found that for aGGM networks, $\lambda_{2}>\lambda_{1}$ in 2998 out of 3000 simulation iterations across all SNR. 
Using the aGGM and cGGM networks we estimated sensitivity and false discovery rate (FDR). Further, we estimated edge weight correlation by correlating edge weights from the reconstructed partial coherence (from cGGM) and amplitude partial correlation (from aGGM) to the edge weights from the ground truth partial coherence and partial correlation. We found that sensitivity, false discovery rate and correlation for both the cGGM and aGGM increased with increasing SNR. We report the average sensitivity, FDR and edge weight correlation results in Table 1, while the full distributions are shown in Figure 4. Sensitivity for the cGGM networks increased from 0.66 (SNR of $1 \mathrm{~dB}$ ) ,i.e. $66 \%$ of SC edges were recovered, to 0.78 (SNR of $50 \mathrm{~dB}$ ) while for the aGGM networks it improved from $0.06(1 \mathrm{~dB})$ to $0.48(50 \mathrm{~dB})$. Remarkably, even at very low signal to noise ratios our simulations show that the cGGM can recover the majority of the SC edges.

False discovery rates for the cGGM decreased from 0.08 (SNR of $1 \mathrm{~dB}$ ), i.e. $8 \%$ of total edges recovered were outside the SC, to 0.03 (SNR of $50 \mathrm{~dB}$ ) and for aGGM it decreased from $0.46(1 \mathrm{~dB})$ to $0.04(50 \mathrm{~dB})$. While reconstruction of network metrics for both cGGM and aGGM improved with SNR, the cGGM networks remained a consistently better predictor of the original network across all metrics (sensitivity, false discovery rate and correlation) at all SNR levels relative to the aGGM networks. Remarkably, even at very low SNR, very few false edges are estimated by the cGGM.

Finally, we observed that sensitivity shows a non-monotonic relationship to SNR and we believe this to be a trade-off occurring in the optimization of the AGL as at low SNR recovery of accurate weights is difficult so instead the algorithm optimizes edge recovery. However, as SNR increases, weights are more recoverable allowing the SC-QUIC process to optimize for reduced edges but improved accuracy in weights for edges retained. This is clear when we examine the monotonic increase in correlation of edge weights across SNR (see Table 1).

\subsubsection{Graph Theoretic Measures are Accurately Reconstructed}

For each reconstructed network in simulation we estimated (weighted) degrees, clustering coefficient, eigenvector centrality, closeness centrality and betweenness centrality. We correlated these measures with the estimates from the original networks (prior to forward modeling and source localization) across all nodes using a Spearman rank correlation. For comparison, we correlated the reconstructed edge weights with the original edges weights. Each of these analyses was carried out for the cGGM and aGGM networks. 


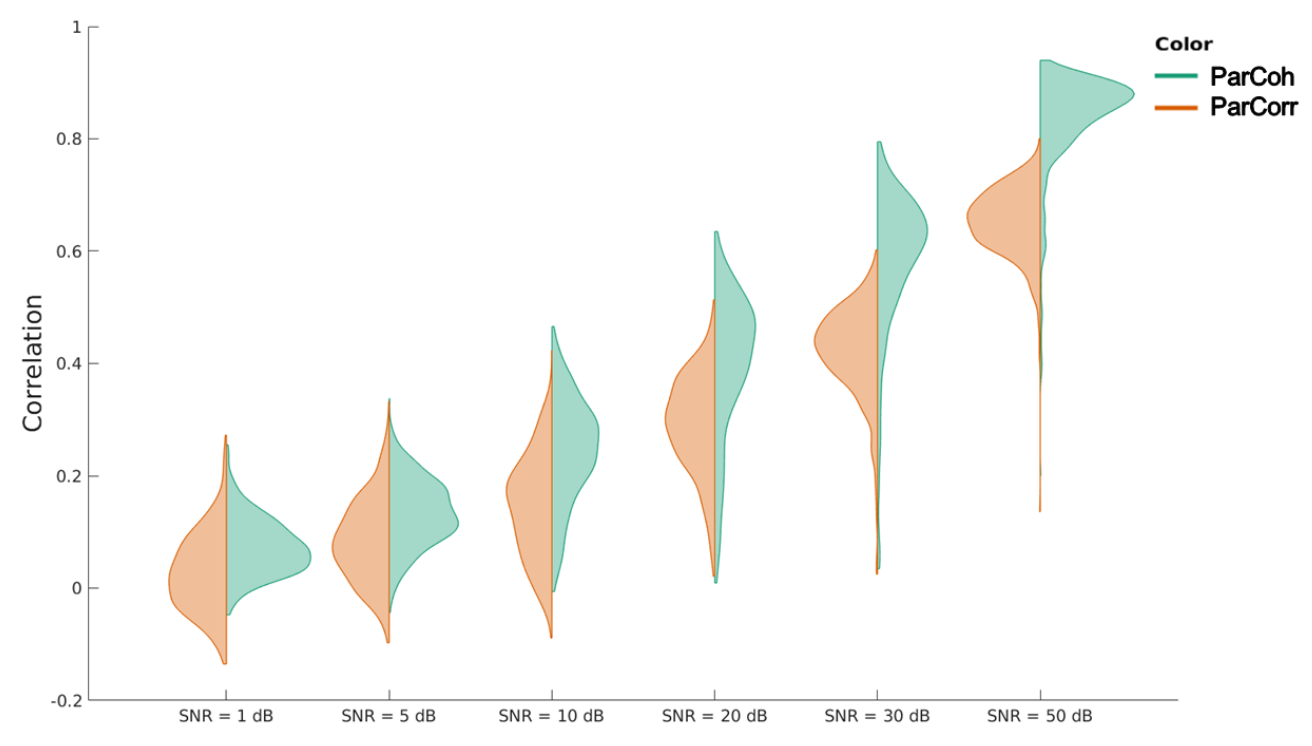

Figure 5: Edge Weight Correlation in Simulation: We estimated networks using the cGGM and aGGM at different signal to noise ratios. We compared the reconstructed partial coherence (cGGM) and partial correlation (aGGM) to the ground truth by examining the correlation in edge weights (over the set of non-zero edges). Partial coherence is more correlated to the ground truth partial coherence than the partial correlation is to the ground truth partial correlation across all SNR. Note that partial coherence has a correlation greater than 0.5 for almost all reconstructions at an SNR above $30 \mathrm{~dB}$ while this is true for partial correlation only at $50 \mathrm{~dB}$.

We found that across all metrics for networks estimated by the cGGM there was monotonic improvement in accuracy with SNR, and in particular the degrees, closeness centrality and betweenness centrality show more rapid improvement with SNR. The full distribution of correlations across all simulations is shown in Figure 6. Degrees, closeness centrality and betweenness centrality outperformed the reconstruction reliability of edge weights based on the partial coherence over all SNR except at $50 \mathrm{~dB}$. The range of correlation for degrees was: 0.32 at $1 \mathrm{~dB}$ to 0.73 at $50 \mathrm{~dB}$, for closeness centrality it was 0.34 to 0.8 and for betweenness centrality it was: 0.31 at $1 \mathrm{~dB}$ to 0.72 at $50 \mathrm{~dB}$. In contrast, we found that the correlation in edge weights lay within a range of 0.07 at $1 \mathrm{~dB}$ to 0.83 at $50 \mathrm{~dB}$. Eigenvector centrality was comparable or slightly worse at some SNR (1 to $20 \mathrm{~dB}$ ) to the reliability of the network edge weight reconstruction while clustering coefficient was inaccurately reconstructed. We show the average correlations for all graph theory metrics 


\begin{tabular}{|c|c|c|c|c|c|c|c|c|c|c|c|c|}
\hline & \multicolumn{2}{|c|}{$1 \mathrm{~dB}$} & \multicolumn{2}{|c|}{$5 \mathrm{~dB}$} & \multicolumn{2}{|c|}{$10 \mathrm{~dB}$} & \multicolumn{2}{|c|}{$20 \mathrm{~dB}$} & \multicolumn{2}{|c|}{$30 \mathrm{~dB}$} & \multicolumn{2}{|c|}{$50 \mathrm{~dB}$} \\
\hline & PC & APC & $\mathrm{PC}$ & APC & $\mathrm{PC}$ & APC & PC & APC & PC & APC & PC & APC \\
\hline Sensitivity & 0.66 & 0.06 & 0.65 & 0.09 & 0.56 & 0.08 & 0.64 & 0.14 & 0.76 & 0.24 & 0.78 & 0.48 \\
\hline FDR & 0.08 & 0.46 & 0.08 & 0.4 & 0.05 & 0.33 & 0.05 & 0.16 & 0.05 & 0.1 & 0.03 & 0.04 \\
\hline Edge Correlation & 0.07 & 0.03 & 0.14 & 0.08 & 0.25 & 0.14 & 0.4 & 0.29 & 0.58 & 0.42 & 0.83 & 0.65 \\
\hline Betweenness Centrality & 0.31 & 0.25 & 0.36 & 0.29 & 0.4 & 0.3 & 0.48 & 0.35 & 0.56 & 0.38 & 0.72 & 0.5 \\
\hline Closeness Centrality & 0.34 & 0.24 & 0.41 & 0.3 & 0.47 & 0.35 & 0.56 & 0.42 & 0.66 & 0.48 & 0.8 & 0.55 \\
\hline Clustering Coefficient & 0.15 & 0.1 & 0.17 & 0.11 & 0.2 & 0.12 & 0.18 & 0.15 & 0.17 & 0.15 & 0.23 & 0.13 \\
\hline Degrees & 0.32 & 0.18 & 0.35 & 0.22 & 0.41 & 0.23 & 0.49 & 0.28 & 0.58 & 0.32 & 0.73 & 0.4 \\
\hline Eigenvector Centrality & 0.21 & 0.21 & 0.27 & 0.23 & 0.32 & 0.27 & 0.39 & 0.31 & 0.47 & 0.34 & 0.57 & 0.37 \\
\hline
\end{tabular}

Table 1: Simulation Results: We show the average for different reconstruction performance metrics and for different summary statistics across all 500 simulation iterations. Each column represents the results for a particular summary statistic (partial coherence - PC or amplitude partial correlation - APC) at a particular signal to noise ratio (1 to $50 \mathrm{~dB})$. The third row shows the average correlations between edge weights from estimated and ground truth PC/APC. The fourth row onward shows the correlation between estimated and ground truth graph theoretic metrics for PC and APC. For full distributions of results across all 500 simulation iterations see Figures 456 , and 7 .

and the average correlation in edge weights in Table 1.

When examining the same graph theory metrics for the aGGM networks we found similar results to what we saw using cGGM networks. For aGGM networks, we compared the graph theory metrics calculated from the original partial correlation (prior to forward modeling and source localization) to the reconstructed partial correlation. All graph theory metrics improved with increasing SNR (see Figure 7). Closeness centrality and betweenness centrality were more (or equally as) accurate estimators of the original network than partial correlation edge weights across all SNR below $50 \mathrm{~dB}$. Degrees and eigenvector centrality were better only at SNR below $20 \mathrm{~dB}$, while clustering coefficient was a better estimate below $10 \mathrm{~dB}$ SNR. The range of correlation (averaged over simulations at each SNR) for degrees was: 0.18 at $1 \mathrm{~dB}$ to 0.4 at $50 \mathrm{~dB}$, for closeness centrality it was 0.24 at $1 \mathrm{~dB}$ to 0.55 at $50 \mathrm{~dB}$ and for betweenness centrality it was: 0.25 at $1 \mathrm{~dB}$ to 0.5 at $50 \mathrm{~dB}$. In contrast, we found that the correlation in edge weights followed a range of 0.03 at $1 \mathrm{~dB}$ to 0.65 at $50 \mathrm{~dB}$. Thus, graph theory metrics were more accurately reconstructed than edge weights for both the cGGM and aGGM networks, but, the benefit in accuracy is reduced at higher SNR. 

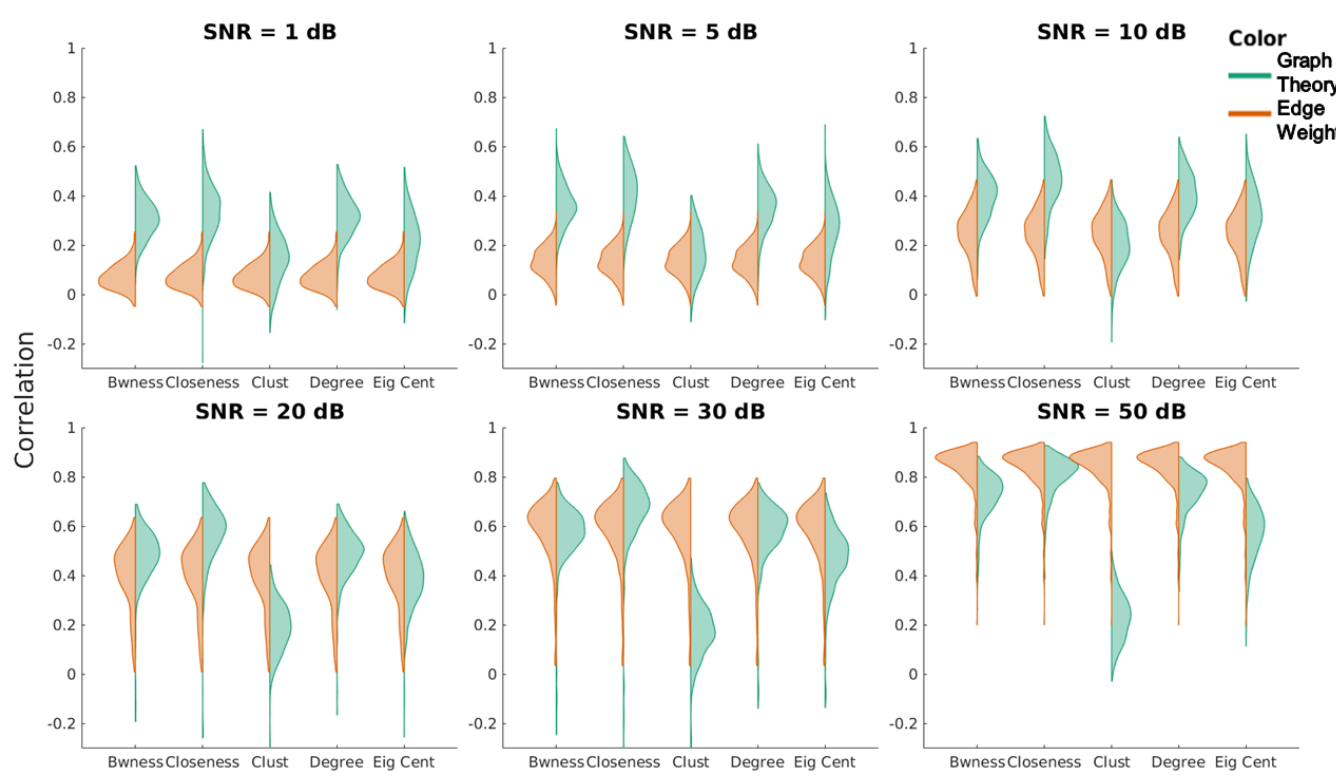

Figure 6: Graph Theory and Partial Coherence: We show how well using different graph theoretic metrics does at capturing the structural connectivity characteristics relative to using the reconstructed partial coherence weights. At each SNR, we plot the correlation between graph theory metrics calculated from reconstructed network with the original network across 500 iterations in green. In orange we show the correlation of the weights of reconstructed partial coherence with the original partial coherence. For comparison purposes we show the same set of correlations (of edge weights) alongside all graph theory metrics. We examine the two methods across different SNR and find that at SNR below $50 \mathrm{~dB}$, closeness centrality, betweenness centrality and degrees provide more accurate reconstruction.

\section{2. $M E G$ Data}

We extracted 480 seconds of preprocessed resting state MEG data from 91 subjects from the open source CAMCAN dataset. We source localized this data to the 114 sources of the Lausanne parcellation before using the AGL and our cross-validation procedure (see section 2.2.5 on Cross Validation in Methods) to estimate the most representative aGGM and cGGM models in each of five frequency bands - delta, theta, alpha, beta and gamma bands.

We found that for the aGGM and cGGM models for 91 subjects, the majority of models built indicated that using the prior of the SC was useful (i.e. in Equation $12 \lambda_{2}>\lambda_{1}$ ). We saw in aGGM models built from CV that the penalization outside the $\mathrm{SC}\left(\lambda_{2}\right)$ was greater than that inside the SC $\left(\lambda_{1}\right)$ for 88 subjects in delta, 89 subjects in theta, 89 subjects in alpha, 

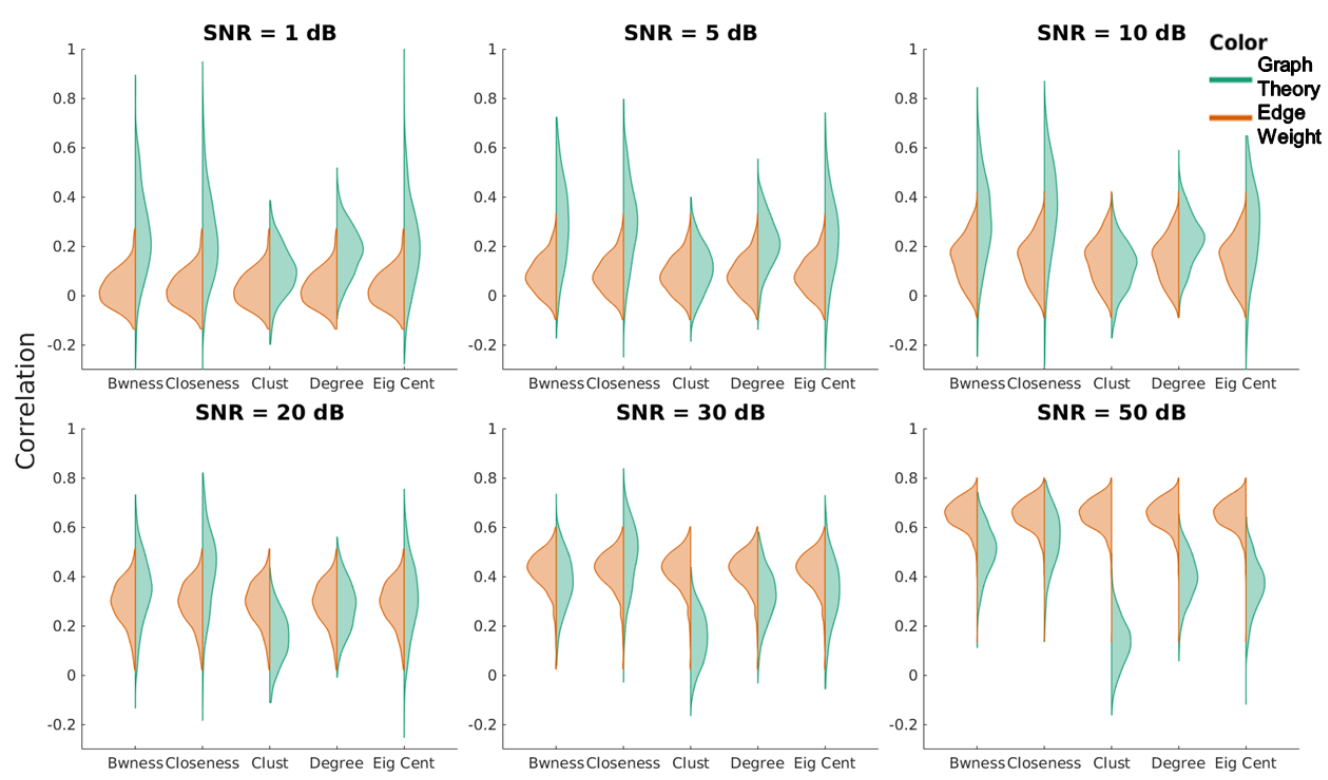

Figure 7: Graph Theory and Amplitude Partial Correlation: We show how well different graph theoretic metrics can capture the structural connectivity characteristics relative to using the reconstructed partial correlation weights. At each SNR, we plot the correlation between graph theory metrics calculated from reconstructed network with the original network across 500 iterations in green. In orange we show the correlation of the weights of reconstructed partial correlation with the original partial correlation. For comparison purposes we show the same set of correlations alongside all graph theory metrics. We examine the two methods across different SNR and find that at SNR below $30 \mathrm{~dB}$, closeness centrality, betweenness centrality and degrees provide more accurate reconstruction.

91 subjects in beta and 90 subjects in gamma. For the cGGM models built based on $\mathrm{CV}$, we saw that the penalization applied was greater outside the $\mathrm{SC}$ in 66 subjects in delta, 75 subjects in theta band, 74 subjects in alpha band, 85 subjects in beta band and 89 subjects in gamma band. For all models we estimated the summary statistics of partial coherence (PC) and amplitude partial correlation (APC). We also estimated graph theory metrics we identified as most accurately reconstructed from our simulation - weighted degrees, closeness centrality and betweenness centrality.

\subsubsection{Alignment of $S C$ to $c G G M$ and aGGM models}

We estimated partial coherence and amplitude partial correlation for all five frequency bands and estimated the sensitivity and false discovery rate (See Figure 8). Sensitivity is informative about the number of edges within 
the SC that were discovered while FDR tells us how often edges outside the SC were a part of the graphical model. Note that since we use crossvalidation to determine penalization, sensitivity and FDR are informative about how useful the SC prior is, and by extension, the relevance of the SC to effective connectivity. We found that on average the sensitivity across subjects when networks were estimated with cGGM were: Delta (mean \pm standard deviation) $-0.09 \pm 0.14$, Theta - 0.15 \pm 0.16 , Alpha - $0.18 \pm 0.19$, Beta $-0.42 \pm 0.26$ and Gamma - $0.75 \pm 0.25$. This indicates that, of the 720 edges in the SC, on average across subjects we identified 540 edges in the gamma band cGGM. When using aGGM on the other hand we found sensitivity across bands was: Delta (mean \pm standard deviation) $-.21 \pm .18$ , Theta $-0.27 \pm 0.22$, Alpha $-0.48 \pm 0.25$, Beta $0.57 \pm 0.21$, and Gamma $-0.45 \pm 0.19$. So in the beta band, we were able to, on average, estimate $57 \%$ of the SC connections, i.e. 410 edges. Sensitivity in aGGM compared to sensitivity in cGGM was significantly higher across delta, theta, alpha and beta bands (Wilcoxon rank-sum test, $p<0.001$ ) but in gamma band it was significantly lower (Wilcoxon rank-sum test, $p<0.001$ ). So at lower frequencies $(<30 \mathrm{~Hz})$ amplitude partial correlation has higher sensitivity to the SC, but at higher frequencies, partial coherence has considerably higher sensitivity. Importantly, the single measure with the highest sensitivity to the $S C$ was the gamma band cGGM which incorporates phase information.

We also examined the false discovery rate (see Figure 8) and found that beta and gamma band in cGGM had the lowest FDR. In partial coherence FDR for different bands were: Delta (mean \pm standard deviation) $-0.48 \pm$ 0.26 , Theta $-0.37 \pm 0.25$, Alpha - $0.4 \pm 0.27$, Beta - $0.29 \pm 0.22$ and Gamma $-0.28 \pm 0.18$. This indicates that, if in the gamma band we estimated 1000 edges on average, of those 1000 edges 280 edges were found outside the SC. While for aGGM, FDR for different bands were: Delta (mean \pm standard deviation) - $0.32 \pm 0.25$, Theta - $0.3 \pm 0.19$, Alpha - $0.28 \pm 0.22$, Beta $0.32 \pm 0.19$ and Gamma - $0.4 \pm 0.22$. False discovery rate measured on real data is incorporating potential connections due to subcortical structural connections and individual specific structural connections not present in our $\mathrm{SC}$.

\subsubsection{Centrality}

From our simulations we identified that degree, closeness centrality and betweenness centrality were potentially more accurate than directly examining the recovered weights of reconstructed networks. We estimated the 

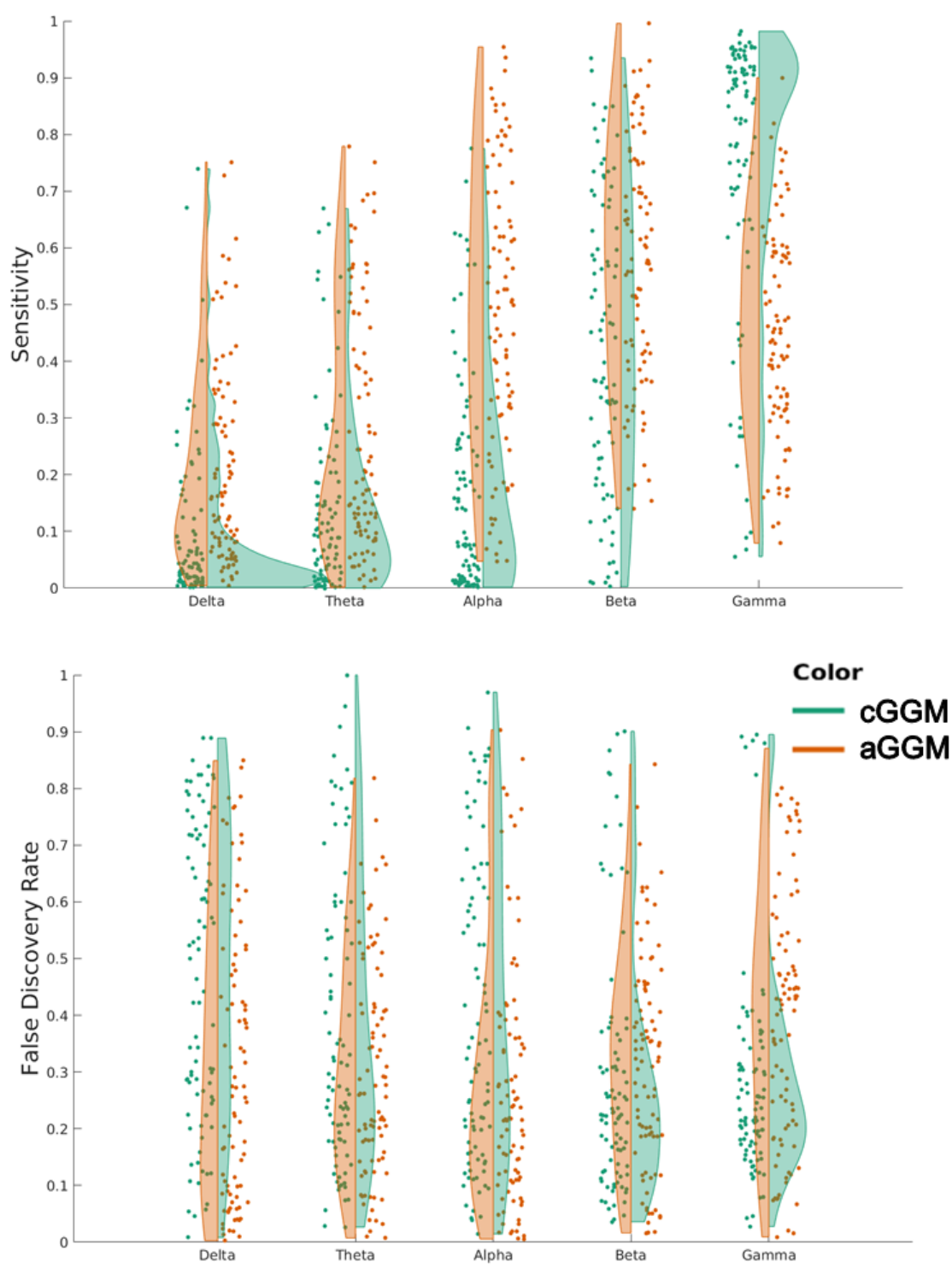

Figure 8: Sensitivity and False Discovery Rate in MEG data: We estimated networks using the cGGM and aGGM at different signal to noise ratios. We compared these networks to the binarized SC and examined the sensitivity (upper figure) and false discovery rate (lower figure) between them. cGGM networks are more sensitive to the SC in the beta and gamma bands while aGGM networks are more sensitive to SC in alpha and beta bands. The cGGM network has lower false discovery rates relative to aGGM networks in beta and gamma bands. 
degree, closeness centrality and betweenness centrality for the aGGM in beta band and for the cGGM in gamma band based on the high sensitivity to the SC in these networks. Taking the median across subjects in each measure and for each set of networks (gamma cGGM, beta aGGM) we estimated Spearman rank correlation to the degrees, closeness centrality and betweenness centrality estimated from the original weighted SC. Similar to our analysis for the simulations, we also correlated the median edge weights for the gamma cGGM and the beta aGGM with the weighted SC. Note that since all networks are weighted and we used cross-validation to estimate penalization, we expect estimates to be divergent between the effective connectivity and the SC. When using the edge weights directly, we found weak but significant correlations between the gamma band edge weights and the SC $(r=0.08, p<0.05)$ and also for the beta band edge weights $(r=0.08, p<0.05)$. In contrast, we saw that the graph theory metrics outperformed edge weight reconstruction. We found that gamma band degrees $(r=0.29, p<0.01)$ and beta band degrees $(r=0.33, p<0.001)$ were significantly correlated to the SC degrees. We show the degrees over areas for the gamma, beta and SC in Figure 9, where it is clear that, while there is a strong tie to the SC centrality, gamma and beta bands support different patterns of connectivity. Beta band has higher degrees in the precentral gyrus and superior parietal areas while in gamma band, we see greater connectivity in frontal areas. Closeness centrality in gamma $(r=0.26, p<0.05)$ band was linked to the SC closeness centrality while beta band only showed a weak relationship $(r=0.17)$. We saw that with $\mathrm{SC}$ betweenness centrality, gamma $(r=0.28, p<0.01)$ and beta band $(r=0.21, p<0.05)$ betweenness centrality showed strong relationships. As expected from our simulations, the correlations of the graph theoretic metrics outperformed the edge weight reconstructions.

\section{Discussion}

\subsection{Modeling the Coherence and the Amplitude Partial Correlation}

Past work examining the link between MEG functional networks and structural connectivity have shown significant, but weak relationships between structure and function. We built models of the MEG coherence using cGGM models and models of the MEG amplitude correlation using the aGGM models. We examined the network recovery from the cross-validated cGGM and aGGM using simulations and showed that at reasonable SNR 

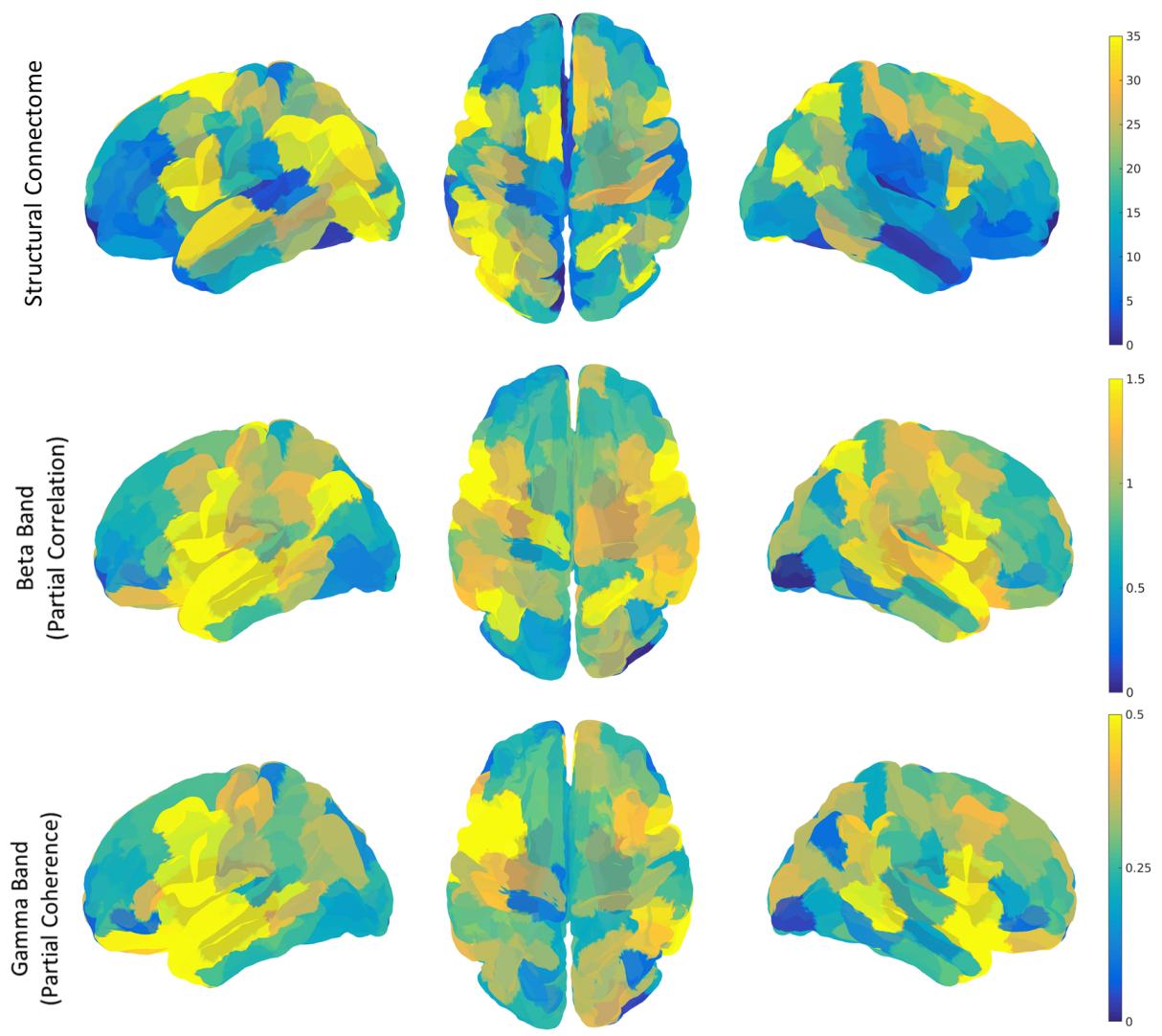

Figure 9: Degrees Across Connectomes: We show degrees across different areas in the structural connectome. Similarly we show median degrees across subjects in the beta band when using partial correlation, and in gamma band when using partial coherence. 
$(>10 \mathrm{~d} B)$, sensitivity above $50 \%$ is possible (implying that field spread and common input effects can be partially overcome in estimation). In MEG data we then identified that, gamma band cGGM reflects the SC more closely than any other network estimate ( $75 \%$ sensitivity, $28 \%$ FDR). This result indicates that models of coherence have substantial information about the connectome, but primarily in higher frequencies. Models of the slower time scale amplitude correlation reflect information about the SC primarily in lower frequencies, reaching a peak in the beta band ( $57 \%$ sensitivity). On average, across all these measures and frequency bands, FDR was around $30 \%$, indicating that a third of the edges recovered were outside the SC. There are three implications of these results:

1. The binarized SC provides a valid and useful blueprint from which to estimate the effective connectivity using partial coherence and partial correlation.

2. There are potentially edges that we have failed to account for because our SC estimate fails to include some genuine anatomical connections

3. Both the coherence and amplitude correlation based networks reflect the SC to varying degrees, with cGGM reflecting the SC more strongly at higher frequencies $(>30 \mathrm{~Hz})$ while aGGM reflects it better at lower frequencies $(1-30 \mathrm{~Hz})$.

Why do the cGGM and aGGM models reflect divergent results? One possibility is that at lower frequencies the uncertainty in phase is much greater than the range of possible phases. For example in the theta band (around $5 \mathrm{~Hz}$ ), at axon conduction velocities of $10 \mathrm{~m} / \mathrm{s}$, even a long axonal fiber of $20 \mathrm{~cm}$ length results in a delay of only $20 \mathrm{~ms}$ or a phase difference in the range of $\pi / 10$. In other words, at low frequencies, the delays within the brain may only be a small fraction of the period of the oscillation. In these cases, leakage effects (which only occur at zero lag) might overwhelm the possibility of being able to estimate genuine phase consistency relationships. In this case the amplitude might serve as a better connectivity estimate by providing greater signal to noise ratios. At higher frequencies however, more of the phase spectrum is accessible, possibly improving the ability to estimate genuine phase relationships. Thus, it is possible that the higher sensitivity of gamma band partial coherence relative to the amplitude partial correlation reflects information in genuine phase relationships in the gamma band resulting from transmission delays across the SC [54]. A second possibility is proposed by [31. They use Kuramoto oscillators (at $40 \mathrm{~Hz}$ ) connected 
by the structural connectome and tuned to maximize fit to empirical amplitude envelope correlations. They show how frequency modulation due to the presence of temporarily synchronized (phase difference of 0) groups of areas (chimera states) could yield the amplitude envelope modulation on the rate of $0.1 \mathrm{~Hz}$ as reported in the literature. This idea even has some empirical backing [55]. Note that given this mechanistic model, coherence in lower frequencies would be too noisy (when we have too few samples) and thus not a robust estimate for connectivity. Another possibility is that lower frequencies simply are not expressed well in the way we have parcelled the brain. It may be a good idea to use a coarse parcellation for lower frequencies given prior knowledge that oscillatory activity in lower frequencies tends to recruit larger groups of neurons [56].

\subsection{Using Graph Theory to Characterize Networks}

Using graph theoretic metrics to summarize networks showed reduced error when using degree, closeness centrality and betweenness centrality over the majority of SNR levels for both PC and APC networks. However, in contrast, clustering coefficient and eigenvector centrality showed increased error in reconstructed networks relative to other graph theoretic metrics. We estimated degree, closeness centrality and betweenness centrality for the gamma band MEG-PC, and the alpha and beta band MEG-APC. We found significant correlations between all centrality estimates with the SC based estimates, however, there was considerable unexplained variance indicating the hubs in data do not exactly map onto the hubs from the SC. In summary, these results suggest that using graph theory to collapse connectivity structures of nodes is useful and robust but only for a subset of metrics.

Using graph theoretic tools to summarize networks has been suggested and applied in highly specific ways in the literature, such as by examining the minimum spanning tree of a network [57]. The minimum spanning tree (MST) represents the skeletal backbone of the network and the underlying interpretation is that using this approach improves robustness of the inference on the networks. A recent empirical study examined MST properties for networks constructed at the scalp and at source level. They found that for most network characteristics (as estimated using the MST) there was weak correlation between scalp and source level. Other simulation work [58] has shown that identifying the generative process that created a network is difficult to impossible when analyzing scalp based networks. For these reasons estimation of graph theoretic metrics are better done at source level 
given the theoretical and empirical justifications of using source localized data [59]. We expanded on these questions by examining which graph theory tools can be estimated well at source level. Further, we examined whether the accuracy of graph theoretic tools improved on directly using the weight structure of the networks. We have shown that we can in fact identify hub nodes using degree or closeness centrality or betweenness and expect those estimates to be closer to the underlying structural connectome relative to using the weights of the network. However, other graph theory tools such as the clustering coefficient and eigenvector centrality were poor estimates. Clustering coefficient has been shown to be biased (underestimated) when using partial correlation estimates [60] and this may be part of the reason it is a poor estimate.

Our results suggested that maximum accuracy with respect to reconstructing network characteristics in alignment with the SC was possible using the degrees, closeness centrality and betweenness centrality. We estimated these measures for the networks that had the highest sensitivity to the SC from the empirical MEG networks - gamma band partial coherence and beta band partial correlation. We found that for these networks there was a significant correlation to the centrality estimated from the weighted SC. This result indicates that graph theory metrics provide useful information about the network properties in different bands, and are possibly more robust than directly examining edge weights.

\subsection{Limitations}

Several limitations exist in our work. In our simulation we assumed a generative model where different parts of the brain show random oscillatory behavior linked by the structural connectome. This could be represented using a zero mean complex multivariate normal with a circularly symmetric precision. More detailed mean field models of neural activity may be more phenomenologically accurate [31], although, past work suggests there is limited gain in using when explaining empirical data [61, 62]. Another issue is that we assumed a common generative model for both the amplitude and the coherence based networks. Other work suggests that potentially there may be independent mechanisms at play in generating these two modes of neuronal coupling [63, 64, 31].

Source localization can be formulated in several ways based on prior assumptions. While we used a weighted $L^{2}$ norm inverse, beamformer reconstruction approaches are also quite common in MEG [65, 12] and require 
investigation within this framework. Bayesian techniques account for priors more explicitly and afford better source reconstruction [66, 67]. Examining these alternative approaches was beyond our scope. Additionally, we chose to limit our analysis to a case where there are 114 sources, a future extension to this work might examine cases with more (fewer) sources. We also ignore for our purposes subcortical source activity and connectivity. This may have led to the large variation in the estimated results in the MEG data. Estimation of subcortical activity in MEG while possible is difficult without explicit prior knowledge [68], and would also potentially benefit from including the magnetometer recordings.

\subsection{Conclusion}

In summary, we used simulations and data to determine the relevance of the structural connectivity to the statistical structure of MEG signals. Real (aGGM) and complex-valued (cGGM) graphical models were used as models of the amplitude correlation and coherence, respectively. We showed that cGGM is a high sensitivity, low false discovery rate estimate of the SC in simulations. Further, we saw that graph theory can improve accuracy in reconstructing SC characteristics. In data, we found that cGGM models of the gamma band had the highest sensitivity to the SC. Across cGGM and aGGM models of data we found that the false discovery rate hovered around $30 \%$ suggesting that our SC may be incomplete, or there are unaccounted for influences on the effective connectivity, suggesting the model could be improved by using better SC estimates. Finally, we saw that our results using graph theory in simulations were borne out in the data where graph theoretic metrics of gamma band partial coherence and beta band partial correlation showed a stronger correlation to the SC than the edge weight correlation. Our work provides evidence in favor of the relevance of the structural connectome in facilitating statistical dependencies across both milliseconds and seconds timescales in MEG data.

\section{References}

[1] J. Gross, N. Hoogenboom, G. Thut, P. Schyns, S. Panzeri, P. Belin, S. Garrod, Speech rhythms and multiplexed oscillatory sensory coding in the human brain, PLoS biology 11 (2013) e1001752. 
[2] F. Roux, P. J. Uhlhaas, Working memory and neural oscillations: alphagamma versus theta-gamma codes for distinct wm information?, Trends in cognitive sciences 18 (2014) 16-25.

[3] C. J. Stam, Modern network science of neurological disorders, Nature Reviews Neuroscience 15 (2014) 683.

[4] S. Rouhinen, J. Panula, J. M. Palva, S. Palva, Load dependence of $\beta$ and $\gamma$ oscillations predicts individual capacity of visual attention, Journal of Neuroscience 33 (2013) 19023-19033.

[5] S. Baillet, Magnetoencephalography for brain electrophysiology and imaging, Nature neuroscience 20 (2017) 327.

[6] F. Siebenhühner, S. H. Wang, J. M. Palva, S. Palva, Cross-frequency synchronization connects networks of fast and slow oscillations during visual working memory maintenance, Elife 5 (2016) e13451.

[7] J. S. Bendat, A. G. Piersol, Random data: analysis and measurement procedures, volume 729, John Wiley \& Sons, 2011.

[8] P. L. Nunez, R. Srinivasan, Electric fields of the brain: the neurophysics of EEG, Oxford University Press, USA, 2006.

[9] R. Srinivasan, W. R. Winter, J. Ding, P. L. Nunez, Eeg and meg coherence: measures of functional connectivity at distinct spatial scales of neocortical dynamics, Journal of neuroscience methods 166 (2007) $41-52$.

[10] P. Fries, Rhythms for cognition: communication through coherence, Neuron 88 (2015) 220-235.

[11] M. J. Brookes, J. R. Hale, J. M. Zumer, C. M. Stevenson, S. T. Francis, G. R. Barnes, J. P. Owen, P. G. Morris, S. S. Nagarajan, Measuring functional connectivity using meg: methodology and comparison with fcmri, Neuroimage 56 (2011) 1082-1104.

[12] M. J. Brookes, M. Woolrich, H. Luckhoo, D. Price, J. R. Hale, M. C. Stephenson, G. R. Barnes, S. M. Smith, P. G. Morris, Investigating the electrophysiological basis of resting state networks using magnetoencephalography, Proceedings of the National Academy of Sciences 108 (2011) 16783-16788. 
[13] J. F. Hipp, D. J. Hawellek, M. Corbetta, M. Siegel, A. K. Engel, Largescale cortical correlation structure of spontaneous oscillatory activity, Nature neuroscience 15 (2012) 884.

[14] F. De Pasquale, S. Della Penna, A. Z. Snyder, C. Lewis, D. Mantini, L. Marzetti, P. Belardinelli, L. Ciancetta, V. Pizzella, G. L. Romani, et al., Temporal dynamics of spontaneous meg activity in brain networks, Proceedings of the National Academy of Sciences 107 (2010) 6040-6045.

[15] C. J. Honey, R. Kötter, M. Breakspear, O. Sporns, Network structure of cerebral cortex shapes functional connectivity on multiple time scales, Proceedings of the National Academy of Sciences 104 (2007) 1024010245.

[16] M. Straathof, M. R. Sinke, R. M. Dijkhuizen, W. M. Otte, A systematic review on the quantitative relationship between structural and functional network connectivity strength in mammalian brains, Journal of Cerebral Blood Flow \& Metabolism (2018) 0271678X18809547.

[17] P. Garcés, E. Pereda, J. A. Hernández-Tamames, F. Del-Pozo, F. Maestú, J. Ángel Pineda-Pardo, Multimodal description of whole brain connectivity: A comparison of resting state meg, fmri, and dwi, Human brain mapping 37 (2016) 20-34.

[18] P. Tewarie, A. Hillebrand, E. van Dellen, M. M. Schoonheim, F. Barkhof, C. Polman, C. Beaulieu, G. Gong, B. W. van Dijk, C. J. Stam, Structural degree predicts functional network connectivity: a multimodal restingstate fmri and meg study, Neuroimage 97 (2014) 296-307.

[19] H.-O. Karnath, C. Sperber, C. Rorden, Mapping human brain lesions and their functional consequences, Neuroimage 165 (2018) 180-189.

[20] K. H. Maier-Hein, P. F. Neher, J.-C. Houde, M.-A. Côté, E. Garyfallidis, J. Zhong, M. Chamberland, F.-C. Yeh, Y.-C. Lin, Q. Ji, et al., The challenge of mapping the human connectome based on diffusion tractography, Nature communications 8 (2017) 1349.

[21] A. Wodeyar, R. Srinivasan, Network structure during encoding predicts working memory performance, bioRxiv (2018) 409615. 
[22] R. Dahlhaus, Graphical interaction models for multivariate time series, Metrika 51 (2000) 157-172.

[23] J. A. Pineda-Pardo, R. Bruña, M. Woolrich, A. Marcos, A. C. Nobre, F. Maestú, D. Vidaurre, Guiding functional connectivity estimation by structural connectivity in meg: an application to discrimination of conditions of mild cognitive impairment, Neuroimage 101 (2014) 765777.

[24] A. Fornito, A. Zalesky, E. Bullmore, Fundamentals of brain network analysis, Academic Press, 2016.

[25] E. Bullmore, O. Sporns, The economy of brain network organization, Nature Reviews Neuroscience 13 (2012) 336.

[26] J. Goñi, M. P. van den Heuvel, A. Avena-Koenigsberger, N. V. de Mendizabal, R. F. Betzel, A. Griffa, P. Hagmann, B. Corominas-Murtra, J.P. Thiran, O. Sporns, Resting-brain functional connectivity predicted by analytic measures of network communication, Proceedings of the National Academy of Sciences 111 (2014) 833-838.

[27] B. Mišić, R. F. Betzel, A. Nematzadeh, J. Goñi, A. Griffa, P. Hagmann, A. Flammini, Y.-Y. Ahn, O. Sporns, Cooperative and competitive spreading dynamics on the human connectome, Neuron 86 (2015) $1518-1529$.

[28] F.-C. Yeh, S. Panesar, D. Fernandes, A. Meola, M. Yoshino, J. C. Fernandez-Miranda, J. M. Vettel, T. Verstynen, Population-averaged atlas of the macroscale human structural connectome and its network topology, NeuroImage 178 (2018) 57-68.

[29] L. Cammoun, X. Gigandet, D. Meskaldji, J. P. Thiran, O. Sporns, K. Q. Do, P. Maeder, R. Meuli, P. Hagmann, Mapping the human connectome at multiple scales with diffusion spectrum mri, Journal of neuroscience methods 203 (2012) 386-397.

[30] R. Srinivasan, P. L. Nunez, R. B. Silberstein, Spatial filtering and neocortical dynamics: estimates of eeg coherence, IEEE Transactions on Biomedical Engineering 45 (1998) 814-826. 
[31] J. Cabral, H. Luckhoo, M. Woolrich, M. Joensson, H. Mohseni, A. Baker, M. L. Kringelbach, G. Deco, Exploring mechanisms of spontaneous functional connectivity in meg: how delayed network interactions lead to structured amplitude envelopes of band-pass filtered oscillations, Neuroimage 90 (2014) 423-435.

[32] D. C. Van Essen, S. M. Smith, D. M. Barch, T. E. Behrens, E. Yacoub, K. Ugurbil, W.-M. H. Consortium, et al., The wu-minn human connectome project: an overview, Neuroimage 80 (2013) 62-79.

[33] M. Cieslak, Easy lausanne, 2015.

[34] J. B. Colby, L. Soderberg, C. Lebel, I. D. Dinov, P. M. Thompson, E. R. Sowell, Along-tract statistics allow for enhanced tractography analysis, Neuroimage 59 (2012) 3227-3242.

[35] P. Hagmann, L. Cammoun, X. Gigandet, R. Meuli, C. J. Honey, V. J. Wedeen, O. Sporns, Mapping the structural core of human cerebral cortex, PLoS biology 6 (2008) e159.

[36] P. J. Schreier, L. L. Scharf, Statistical signal processing of complexvalued data: the theory of improper and noncircular signals, Cambridge University Press, 2010.

[37] P. Malmivuo, J. Malmivuo, R. Plonsey, Bioelectromagnetism: principles and applications of bioelectric and biomagnetic fields, Oxford University Press, USA, 1995.

[38] B. Fischl, Freesurfer, Neuroimage 62 (2012) 774-781.

[39] A. Gramfort, T. Papadopoulo, E. Olivi, M. Clerc, Openmeeg: opensource software for quasistatic bioelectromagnetics, Biomedical engineering online 9 (2010) 45 .

[40] A. Dale, Sereno mi improved localization of cortical activity by combining eeg and meg with mri cortical surface reconstruction: A linear approach, J Cogn Neurosci 5 (1993) 162-170.

[41] J. Friedman, T. Hastie, R. Tibshirani, Sparse inverse covariance estimation with the graphical lasso, Biostatistics 9 (2008) 432-441. 
[42] P. Menéndez, Y. A. Kourmpetis, C. J. ter Braak, F. A. van Eeuwijk, Gene regulatory networks from multifactorial perturbations using graphical lasso: application to the dream4 challenge, PloS one 5 (2010) e14147.

[43] G. Varoquaux, A. Gramfort, J.-B. Poline, B. Thirion, Brain covariance selection: better individual functional connectivity models using population prior, in: Advances in neural information processing systems, pp. 2334-2342.

[44] S. Ryali, T. Chen, K. Supekar, V. Menon, Estimation of functional connectivity in fmri data using stability selection-based sparse partial correlation with elastic net penalty, NeuroImage 59 (2012) 3852-3861.

[45] T. Zerenner, P. Friederichs, K. Lehnertz, A. Hense, A gaussian graphical model approach to climate networks, Chaos: An Interdisciplinary Journal of Nonlinear Science 24 (2014) 023103.

[46] N. Meinshausen, P. Bühlmann, et al., High-dimensional graphs and variable selection with the lasso, The annals of statistics 34 (2006) $1436-1462$.

[47] C.-J. Hsieh, I. S. Dhillon, P. K. Ravikumar, M. A. Sustik, Sparse inverse covariance matrix estimation using quadratic approximation, in: Advances in neural information processing systems, pp. 2330-2338.

[48] H. Zou, The adaptive lasso and its oracle properties, Journal of the American statistical association 101 (2006) 1418-1429.

[49] K. Murphy, M. Dunham, Pmtk: Probabilistic modeling toolkit, in: Neural Information Processing Systems (NIPS) Workshop on Probabilistic Programming.

[50] M. Rubinov, O. Sporns, Complex network measures of brain connectivity: uses and interpretations, Neuroimage 52 (2010) 1059-1069.

[51] J. R. Taylor, N. Williams, R. Cusack, T. Auer, M. A. Shafto, M. Dixon, L. K. Tyler, R. N. Henson, et al., The cambridge centre for ageing and neuroscience (cam-can) data repository: structural and functional mri, meg, and cognitive data from a cross-sectional adult lifespan sample, Neuroimage 144 (2017) 262-269. 
[52] M. A. Shafto, L. K. Tyler, M. Dixon, J. R. Taylor, J. B. Rowe, R. Cusack, A. J. Calder, W. D. Marslen-Wilson, J. Duncan, T. Dalgleish, et al., The cambridge centre for ageing and neuroscience (cam-can) study protocol: a cross-sectional, lifespan, multidisciplinary examination of healthy cognitive ageing, BMC neurology 14 (2014) 204.

[53] C. J. Chu, M. A. Kramer, J. Pathmanathan, M. T. Bianchi, M. B. Westover, L. Wizon, S. S. Cash, Emergence of stable functional networks in long-term human electroencephalography, Journal of Neuroscience 32 (2012) 2703-2713.

[54] J. M. Palva, S. H. Wang, S. Palva, A. Zhigalov, S. Monto, M. J. Brookes, J.-M. Schoffelen, K. Jerbi, Ghost interactions in meg/eeg source space: A note of caution on inter-areal coupling measures, Neuroimage 173 (2018) 632-643.

[55] S. Nelli, S. Itthipuripat, R. Srinivasan, J. T. Serences, Fluctuations in instantaneous frequency predict alpha amplitude during visual perception, Nature communications 8 (2017) 2071.

[56] G. Buzsaki, Rhythms of the Brain, Oxford University Press, 2006.

[57] M. Lai, M. Demuru, A. Hillebrand, M. Fraschini, A comparison between scalp-and source-reconstructed eeg networks, Scientific reports 8 (2018) 12269.

[58] L. Antiqueira, F. A. Rodrigues, B. C. van Wijk, L. d. F. Costa, A. Daffertshofer, Estimating complex cortical networks via surface recordings?a critical note, Neuroimage 53 (2010) 439-449.

[59] J.-M. Schoffelen, J. Gross, Source connectivity analysis with meg and eeg, Human brain mapping 30 (2009) 1857-1865.

[60] A. Zalesky, A. Fornito, E. Bullmore, On the use of correlation as a measure of network connectivity, Neuroimage 60 (2012) 2096-2106.

[61] H. Finger, M. Bönstrup, B. Cheng, A. Messé, C. Hilgetag, G. Thomalla, C. Gerloff, P. König, Modeling of large-scale functional brain networks based on structural connectivity from dti: comparison with eeg derived phase coupling networks and evaluation of alternative methods along the modeling path, PLoS computational biology 12 (2016) e1005025. 
[62] A. Messé, D. Rudrauf, A. Giron, G. Marrelec, Predicting functional connectivity from structural connectivity via computational models using mri: an extensive comparison study, NeuroImage 111 (2015) 65-75.

[63] M. Siems, M. Siegel, Dissociated cortical phase-and amplitude-coupling patterns in the human brain, bioRxiv (2018) 485599.

[64] A. K. Engel, C. Gerloff, C. C. Hilgetag, G. Nolte, Intrinsic coupling modes: multiscale interactions in ongoing brain activity, Neuron 80 (2013) 867-886.

[65] J. Groß, J. Kujala, M. Hämäläinen, L. Timmermann, A. Schnitzler, R. Salmelin, Dynamic imaging of coherent sources: studying neural interactions in the human brain, Proceedings of the National Academy of Sciences 98 (2001) 694-699.

[66] D. Wipf, S. Nagarajan, A unified bayesian framework for meg/eeg source imaging, NeuroImage 44 (2009) 947-966.

[67] S. Baillet, J. C. Mosher, R. M. Leahy, Electromagnetic brain mapping, IEEE Signal processing magazine 18 (2001) 14-30.

[68] P. Krishnaswamy, G. Obregon-Henao, J. Ahveninen, S. Khan, B. Babadi, J. E. Iglesias, M. S. Hämäläinen, P. L. Purdon, Sparsity enables estimation of both subcortical and cortical activity from meg and eeg, Proceedings of the National Academy of Sciences 114 (2017) E10465-E10474. 